\title{
Large Scale CO Observations of a Far-Infrared Loop in Pegasus; Detection of a Large Number of Very Small Molecular Clouds Possibly Formed via Shocks
}

\author{
H. Yamamoto ${ }^{1}$, A. Kawamura ${ }^{1}$, K. Tachihara ${ }^{2}$, N. Mizuno ${ }^{1}$, T. Onishi ${ }^{1}$ and Y. Fukui ${ }^{1}$
}

\begin{abstract}
We have carried out large scale CO observations with a $\mathrm{mm} / \mathrm{sub}-\mathrm{mm}$ telescope NANTEN toward a far infrared loop-like structure whose angular extent is about $20 \times 20$ degrees around $(l, b) \sim\left(109^{\circ},-45^{\circ}\right)$ in Pegasus. Its diameter corresponds to $\sim 25 \mathrm{pc}$ at a distance of $100 \mathrm{pc}$, adopted from that of a star HD886 (B2IV) near the center of the loop. We covered the loop-like structure in the ${ }^{12} \mathrm{CO}(J=$ $1-0)$ emission at $4^{\prime}-8^{\prime}$ grid spacing and in the ${ }^{13} \mathrm{CO}(J=1-0)$ emission at $2^{\prime}$ grid spacing for the ${ }^{12} \mathrm{CO}$ emitting regions. The ${ }^{12} \mathrm{CO}$ distribution is found to consist of 78 small clumpy clouds whose masses range from $0.04 M_{\odot}$ to $11 M_{\odot}$, and $\sim 83 \%$ of the ${ }^{12} \mathrm{CO}$ clouds have very small masses less than $1.0 \mathrm{M} \odot \odot \cdot{ }^{13} \mathrm{CO}$ observations revealed that 18 of the $78{ }^{12} \mathrm{CO}$ clouds show significant ${ }^{13} \mathrm{CO}$ emission. ${ }^{13} \mathrm{CO}$ emission was detected in the region where the column density of $\mathrm{H}_{2}$ derived from ${ }^{12} \mathrm{CO}$ is greater than $5 \times 10^{20} \mathrm{~cm}^{-2}$, corresponding to $A \mathrm{v}$ of $\sim 1 \mathrm{mag}$, which takes into account that of $\mathrm{HI}$. We find no indication of star formation in these clouds in IRAS Point Source Catalog and 2MASS Point Source Catalog. The very low mass clouds, $M \leq 1 M_{\odot}$, identified are unusual in the sense that they have very weak ${ }^{12} \mathrm{CO}$ peak temperature of $0.5 \mathrm{~K}-2.7 \mathrm{~K}$ and that they aggregate in a region of a few pc with no main massive clouds; contrarily to this, similar low mass clouds less than $1 M_{\odot}$ in other regions previously observed including those at high Galactic latitude are all associated with more massive main clouds of $\sim 100 M_{\odot}$. A comparison with a theoretical work on molecular cloud formation (Koyama \& Inutsuka 2002) suggests that the very low-mass clouds may have been formed in the shocked layer through the thermal instability. The star HD886 (B2IV) may be the source of the mechanical luminosity via stellar winds to create shocks, forming the loop-like structure where the very low-mass clouds are embedded.
\end{abstract}

\footnotetext{
${ }^{1}$ Department of Astrophysics, Nagoya University, Chikusa-ku, Nagoya, Japan 464-8602; hiro@a.phys.nagoya-u.ac.jp

${ }^{2}$ Graduate School of Science and Technology, Kobe University, 1-1 Rokko-dai, Nada-ku, Kobe, Japan $657-8501$
} 
Subject headings: ISM: clouds — ISM: individual(High Latitude Clouds) — radio lines: ISM — stars: formation — stars: winds

\section{INTRODUCTION}

High Galactic latitude molecular clouds (hereafter HLCs) are typically located at $|\mathrm{b}|$ $\gtrsim 20^{\circ}-30^{\circ}$. Since the Gaussiun scale height of $\mathrm{CO}$ is estimated to be $\sim 100 \mathrm{pc}$ in the inner Galactic disk (e.g., Magnani et al. 2000), HLCs are likely located very close to the Sun, within a few hundred pc or less. Their proximity to the Sun and the low possibility of overlapping with other objects along the line of sight enable us to study them with a high spatial resolution and to compare $\mathrm{CO}$ data unambiguously with the data at other wavelengths. HLCs have lower molecular densities compared with dark clouds where the optical obscuration is significant. Therefore, HLCs are often called as translucent clouds (e.g., van Dishoeck \& Black 1988) and most of the known HLCs are not the sites of active star formation, although a few of them are known to be associated with T Tauri stars (e.g., Magnani et al. 1995; Pound 1996; Hearty et al. 1999).

Given the very small distances of HLCs, it is a challenging task for observers to make a complete survey for HLCs over a significant portion of the whole sky. ${ }^{12} \mathrm{CO}(J=1-$ 0 ) emission has been used to search for HLCs because the line emission in the mm band is strongest among the thermally or sub-thermally excited spectral lines of interstellar molecular species. It is however difficult to cover an area as large as tens of square degrees subtended

by some of the HLCs because of the general weakness of the ${ }^{12} \mathrm{CO}$ emission, typically $\sim$ a few K (e.g., Magnani et al. 1996), with existing mm-wave telescopes in a reasonable time scale. HLCs have been therefore searched for by employing various large-scale datasets at other wavelengths including the optical obscuration (Magnani et al. 1985; Keto \& Myers 1986), the infrared radiation (Reach et al. 1994), and the far-infrared excess over HI (=FIR excess)(Blitz et al. 1990; Onishi et al. 2001). On the other hand, unbiased surveys in CO at high Galactic latitudes have been performed at very coarse grid separations of $1^{\circ}$ resulting in a small sampling factor of a few \% (Hartmann et al. 1998; Magnani et al. 2000). Most recently, Onishi et al. (2001) discovered 32 HLCs or HLC complexes. This search was made based on the FIR excess, demonstrating the correlation among FIR excess clouds with CO clouds is a useful indicator of CO HLCs.

Previous CO observations of individual HLCs at higher angular resolutions show that HLCs exhibit often loop-like or shell-like distributions having filamentary features with widths of several arc min or less (Hartmann et al. 1998; Magnani et al. 2000; Bhatt 2000), and in addition that HLCs often compose a group, whose angular extent is $\sim 10$ degrees or 
larger. In order to better understand the structure of HLCs and to pursue the evolution of HLC complexes, CO observations covering tens of square degrees at a high angular resolution are therefore crucial. The past observations of such complexes of HLCs are limited to a few regions including Polaris flare (Heithausen \& Thaddeus 1990), Ursa Major (Pound \& Goodman 1997) and the HLC complex toward MBM 53, 54, and 55 (Yamamoto et al. 2003). Pound \& Goodman (1997) showed an arc-like structure of the molecular cloud system and suggested that the origin of such structures could be some explosive events. Most recently, Yamamoto et al. (2003) carried out extensive observations of the molecular cloud complex including MBM 53, 54, and 55 and suggest that the HLCs may be significantly affected by past explosive events based on the arc-like morphologies of molecular hydrogen (see also Gir et al. 1994).

The region of MBM 53, 54, and 55 is of particular interest among the three, because it is associated with a large $\mathrm{HI}$ cloud of $\sim 590 M_{\odot}$ at a latitude of -35 degrees and because there is a newly discovered HLC of $330 M_{\odot}$, HLCG92-35, which is significantly H I rich with a mass ratio $M\left(\mathrm{H}_{2}\right) / M(\mathrm{HI})$ of $\sim 1$, among the known HLCs (Yamamoto et al. 2003). This cloud was in fact missed in the previous surveys based on optical extinction (Magnani et al. 1985). Subsequent to these observations we became aware of that the region is also very rich in interstellar matter as shown by the $100 \mu \mathrm{m}$ dust features (Kiss et al. 2004). There is a loop-like structure shown at $100 \mu \mathrm{m}$ around $(l, b) \sim\left(109^{\circ},-45^{\circ}\right)$. Toward the center of the loop, an early type star HD886(B2IV) is located and may play a role in creating the loop. Its proper motion is large at a velocity of a few $\mathrm{km} \mathrm{s}^{-1}$, suggesting that the stellar winds of the star might have continued to interact with the surrounding neutral matter over a few tens of pc in $\sim$ a few Myr. Magnani et al. (1985) and Onishi et al. (2001) yet observed only a small part of this region. In order to reveal the large scale $\mathrm{CO}$ distribution of the region, we have carried out observations toward $(l, b) \sim\left(109^{\circ},-45^{\circ}\right)$ by ${ }^{12} \mathrm{CO}(J=1-0)$ and ${ }^{13} \mathrm{CO}(J=1-0)$ with NANTEN 4-meter millimeter/sub-mm telescope of Nagoya University at Las Campanas, Chile. We shall adopt the distance of $100 \mathrm{pc}$ from the sun to the loop-like structure which is equal to the distance of the B2 star in the center of the loop, and is also a typical value for the HLCs.

\section{OBSERVATIONS}

${ }^{12} \mathrm{CO}(J=1-0)$ and ${ }^{13} \mathrm{CO}(J=1-0)$ observations were made with the 4-meter telescope, NANTEN, of Nagoya University at Las Campanas Observatory of Carnegie Institutions of

Washington, Chile. The front-end was an SIS receiver cooled down to $4 \mathrm{~K}$ with a closedcycle helium gas refrigerator (Ogawa et al. 1990). The backend was an acousto-optical 
spectrometer with 2048 channels, and the total bandwidth was $40 \mathrm{MHz}$. The frequency resolution was $35 \mathrm{kHz}$, corresponding to a velocity resolution of $\sim 0.1 \mathrm{~km} \mathrm{~s}^{-1}$. A typical system noise temperature was $\sim 200 \mathrm{~K}(\mathrm{SSB})$ at $115.271 \mathrm{GHz}$ and $\sim 150 \mathrm{~K}(\mathrm{SSB})$ at 110.201 $\mathrm{GHz}$. The half-power beam width was about 2'6, corresponding to $0.076 \mathrm{pc}$ at a distance of 100 pc. The pointing accuracy was better than $20^{\prime \prime}$, as established by radio observations of Jupiter, Venus, and the edge of the Sun in addition to optical observations of stars with a CCD camera attached to the telescope.

The observed region in ${ }^{12} \mathrm{CO}$ was $\sim 240$ square degrees toward the whole area of the looplike structure centered at around $(l, b) \sim\left(109^{\circ},-45^{\circ}\right)$ shown in a $100 \mu \mathrm{m}$ map by Schlegel et al. (1998). First, the ${ }^{12} \mathrm{CO}$ observations were made at a grid spacing of $8^{\prime} \times \cos (b)$ and $8^{\prime}$ in Galactic longitude and latitude, respectively. Then, the regions where the ${ }^{12} \mathrm{CO}$ emission is significantly detected were observed at a grid spacing of $4^{\prime} \times \cos (b)$ and $4^{\prime}$ in Galactic longitude and latitude, respectively. The ${ }^{13} \mathrm{CO}$ observations were made in and around the whole area where the peak temperature of ${ }^{12} \mathrm{CO}$ emission is higher than $2.0 \mathrm{~K}$ at a grid spacing of $2^{\prime} \times \cos (b)$ and $2^{\prime}$ in Galactic longitude and latitude, respectively. The periods of ${ }^{12} \mathrm{CO}$ observations were several sessions between 2002 May and November and those of ${ }^{13} \mathrm{CO}$ were those between 2003 April and August. All the observations were made by frequency switching whose interval is $20 \mathrm{MHz}$, corresponding to $\sim 50 \mathrm{~km} \mathrm{~s}^{-1}$. The integration times per point of ${ }^{12} \mathrm{CO}$ and ${ }^{13} \mathrm{CO}$ observations were typically $\sim 30 \mathrm{~s}$ and $\sim 75 \mathrm{~s}$, respectively, resulting in typical rms noise temperatures per channel of $\sim 0.35 \mathrm{~K}$ and $\sim 0.15 \mathrm{~K}$ in the radiation temperature, $T_{\mathrm{R}}^{*}$, respectively. In reducing the spectral data, we subtracted forthorder polynomials for the emission-free parts in order to ensure a flat spectral baseline. Total numbers of observed points of ${ }^{12} \mathrm{CO}$ and ${ }^{13} \mathrm{CO}$ are 16890 and 3100 , respectively.

We employed a room-temperature blackbody radiator and the sky emission for the intensity calibration. An absolute intensity calibration and the overall check of the whole system were made by observing Orion $\mathrm{KL}\left[\alpha(1950)=5^{\mathrm{h}} 32^{\mathrm{m}} 47 .{ }^{\mathrm{s}} 0, \delta(1950)=-5^{\circ} 24^{\prime} 21^{\prime \prime}\right]$ every 2 hours. We assumed the $T_{\mathrm{R}}^{*}$ of Orion KL to be $65 \mathrm{~K}$ for ${ }^{12} \mathrm{CO}$ and $10 \mathrm{~K}$ for ${ }^{13} \mathrm{CO}$.

\section{RESULTS}

\section{1. ${ }^{12} \mathrm{CO}$ Observation}

\subsubsection{Distribution and Past Detection of ${ }^{12} \mathrm{CO}$ Clouds}

Figure 1 shows the distribution of the velocity-integrated intensity map of ${ }^{12} \mathrm{CO}$ emission. We defined a ${ }^{12} \mathrm{CO}$ cloud as a collection of more than two contiguous observed positions 
whose integrated intensity exceeds $0.77 \mathrm{~K} \mathrm{~km} \mathrm{~s}^{-1}(5 \sigma)$. Based on the definition, we identified 78 molecular clouds in this region. Molecular clouds are concentrated from $(l, b) \sim$ $\left(107^{\circ},-37^{\circ}\right)$ to $\left(116^{\circ},-45^{\circ}\right)$ and around $(l, b) \sim\left(114^{\circ},-52^{\circ}\right)$. Most of the molecular clouds are very small, having size of $\lesssim 1^{\circ}$. Figure 2 shows the distribution of the $\mathrm{CO}$ superposed on the SFD $100 \mu \mathrm{m}$ (Schlegel et al. 1998), which was derived from a composite of the COBE/DIRBE and IRAS/ISSA maps, with the foreground zodiacal light and confirmed point sources removed. CO clouds are distributed along the infrared loop whose diameter is $\sim 25$ pc. We detected little CO emission within the loop-like structure, while toward some of the local peaks of SFD $100 \mu \mathrm{m}$ there is no CO emission.

Figure 3 shows the peak radial velocity distribution derived from the present ${ }^{12} \mathrm{CO}$ data set. The velocity in Figure 3 is derived by a single gaussiun fitting from all CO spectra. The velocity range of the molecular clouds is from $-18.3 \mathrm{~km} \mathrm{~s}^{-1}$ to $0.3 \mathrm{~km} \mathrm{~s}^{-1}$ and there is no systematic large scale velocity gradients.

Some of the molecular clouds have already been known by previous observations. Molecular clouds toward $(l, b) \sim(110.18,-41.23)$ and $\left(117^{\circ} .36,-52^{\circ} .28\right)$ are identified by Magnani et al. (1985) and named as MBM 1 and MBM 2, respectively. DIR117-44 and DIR105-38 identified by Reach et al. (1998) are also identified in CO toward $(l, b) \sim\left(116.5,-44^{\circ} 0\right)$ and $\left(105^{\circ} 0,-38^{\circ} .0\right)$ by Onishi et al. (2001). Magnani et al. (1986) detected CO emission at $(l, b) \sim\left(112^{\circ},-40^{\circ}\right)$. Magnani et al. (2000) also covered this region even though they made observations on a locally Cartesian grid with $1^{\circ}$ (true angle) spacing in longitude and latitude for a beam size of 8!8, they detected CO emission at eight positions of $(l, b) \sim(103.2$, $\left.-38^{\circ} .0\right)$, (103.2, -39.0), (104.4, -39.0), (106.8, -37.0), (108.0, -52.0), (109.5, -51.0), $\left(110^{\circ} 4,-41.0\right)$, and $\left(111^{\circ} 0,-50^{\circ} 0\right)$ in the present region while they missed the present small molecular clouds whose sizes are less than several arc min in Figure 1 due to the coarse grid spacing.

\subsubsection{Physical Properties of ${ }^{12} \mathrm{CO}$ Molecular Clouds}

Seventy-eight ${ }^{12} \mathrm{CO}$ molecular clouds are identified in the present region. For each molecular cloud, $\Delta V$ derived from single Gaussian fitting was from 0.5 to $3.7 \mathrm{~km} \mathrm{~s}^{-1}$, and the radial velocity, $V_{\mathrm{LSR}}$, ranges from -15.7 to $-0.1 \mathrm{~km} \mathrm{~s}^{-1}$. The maximum brightness temperature, $T_{\mathrm{R}}^{*}\left({ }^{12} \mathrm{CO}\right)$ ranges from 0.5 to $5.7 \mathrm{~K}$. The radius of a cloud, $R$, which is defined as the radius of an equivalent circle having the same area, i.e., and $R(\mathrm{pc})=\sqrt{A / \pi}$ where $\mathrm{A}$ is the total cloud surface area within the $5 \sigma$-contour level, ranges from 0.07 to $0.79 \mathrm{pc}$. The peak column density of molecular hydrogen, $N\left(\mathrm{H}_{2}\right)$, in each cloud derived by assuming a conversion factor of $1.0 \times 10^{20} \mathrm{~cm}^{-2} /\left(\mathrm{K} \mathrm{km} \mathrm{s}^{-1}\right.$ ) (Magnani et al. 2000) ranges from $8.0 \times 10^{19}$ 
to $1.7 \times 10^{21} \mathrm{~cm}^{-2}$ with the present detection limit, $7.7 \times 10^{19} \mathrm{~cm}^{-2}$, corresponding to mass detection limit of $0.014 M_{\odot}$. We estimate the molecular mass, $M\left({ }^{12} \mathrm{CO}\right)$, by using the following formula

$$
M\left({ }^{12} \mathrm{CO}\right)=\mu m_{\mathrm{H}} \Sigma\left[D^{2} \Omega N\left(\mathrm{H}_{2}\right)\right],
$$

where $\mu$ is the mean molecular weight, assumed to be 2.8 by taking into account a relative helium abundance of $25 \%$ in mass, $m_{\mathrm{H}}$ is the mass of the atomic hydrogen, $D$ is the distance from the Sun to the molecular clouds, and $\Omega$ is the solid angle subtended by a unit grid spacing of $\left(4^{\prime}\right) \times\left(4^{\prime} \times \cos (b)\right) . M\left({ }^{12} \mathrm{CO}\right)$ ranges from $\sim 0.04$ to $\sim 11 M_{\odot}$ and the total mass of molecular clouds is $\sim 64 M_{\odot}$. These physical properties are listed in Table 1 and the histograms of $T_{\mathrm{R}}^{*}\left({ }^{12} \mathrm{CO}\right), \Delta V, \log (R)$, and $\log \left(N\left(\mathrm{H}_{2}\right)\right)$ of these clouds are shown in Figure 4. Histograms in Figure 4 are divided into three different categories, Usual Cloud (hereafter UC) whose mass is greater than $1 M_{\odot}$, Small Cloud (hereafter SC) whose mass is between 0.1 and $1 M_{\odot}$, and Very Small Cloud (hereafter VSC) whose mass is less than $0.1 M_{\odot}$. It is remarkable that there are a number of molecular clouds having mass less than $1 M_{\odot}$ and that the fractions of SC and VSC are $43 / 78 \sim 55 \%$ and $22 / 78 \sim 28 \%$ in the present region, respectively. In addition, the sizes of $\mathrm{SC}$ and $\mathrm{VSC}$ are equal to or less than $0.1 \mathrm{pc}$. We also note that the peak temperatures of SC and VSC are typically in a range from $0.5 \mathrm{~K}$ to 2.7 $\mathrm{K}$, well below that of $\mathrm{UC}$ in the same region.

\subsection{The Detection and Physical Properties of the ${ }^{13} \mathrm{CO}$ Molecular Clouds}

Figure 5 shows the distribution of the velocity-integrated intensity map of the ${ }^{13} \mathrm{CO}$ emission superposed on the ${ }^{12} \mathrm{CO}$ distribution. The total area of the ${ }^{13} \mathrm{CO}$ observations is $\sim$ 29 square degrees toward 38 of the $78{ }^{12} \mathrm{CO}$ clouds. We observed all of $13 \mathrm{UCs}, 24$ of $43 \mathrm{SCs}$, and 3 of 22 VSCs. We detected ${ }^{13} \mathrm{CO}$ emission at 11 of the $13 \mathrm{UCs}, 8$ of the $24 \mathrm{SCs}$, and none of the $3 \mathrm{VSCs}$, indicating a trend that the ${ }^{13} \mathrm{CO}$ intensity increases with ${ }^{12} \mathrm{CO}$ cloud mass.

A ${ }^{13} \mathrm{CO}$ cloud is defined in the same way as for a ${ }^{12} \mathrm{CO}$ cloud except for the lowest integrated intensity level, $0.3 \mathrm{~K} \mathrm{~km} \mathrm{~s}^{-1}(3 \sigma)$. Based on the definition, we identified $33{ }^{13} \mathrm{CO}$ clouds. For the $33{ }^{13} \mathrm{CO}$ molecular clouds, $\Delta V$ derived from single Gaussian fitting is $\sim 1.5$ $\mathrm{km} \mathrm{s}^{-1}$ and $V_{\mathrm{LSR}}$ of them ranges from -13.1 to $-1.9 \mathrm{~km} \mathrm{~s}^{-1}$. Other physical properties, the maximum brightness temperature, $T_{\mathrm{R}}^{*}\left({ }^{13} \mathrm{CO}\right)$, and $R$ range from 0.3 to $2.3 \mathrm{~K}$ and from 0.04 to $0.21 \mathrm{pc}$, respectively. The physical parameters including the molecular column density and mass (hereafter $M_{\text {LTE }}$ ) are derived on the assumption of local thermodynamic equilibrium (LTE). To derive the column density of molecular hydrogen, the optical depth of ${ }^{13} \mathrm{CO}$ is 
estimated by using the following equations,

$$
\tau\left({ }^{13} \mathrm{CO}\right)=\ln \left[1-\frac{T_{\mathrm{R}}^{*}\left({ }^{13} \mathrm{CO}\right)}{5.29}\left\{\frac{1}{\exp \left(5.29 / T_{\mathrm{ex}}\right)-1}-0.164\right\}^{-1}\right],
$$

where $T_{\text {ex }}$ is the excitation temperature of the $J=1-0$ transition of $\mathrm{CO}$ in $\mathrm{K}$ and was derived from

$$
T_{\mathrm{ex}}=\frac{5.53}{\ln \left\{1+5.53 /\left[T_{\mathrm{R}}^{*}\left({ }^{12} \mathrm{CO}\right)+0.819\right]\right\}} .
$$

$T_{\text {ex }}$ was estimated to be $9.4 \mathrm{~K}$ from our ${ }^{12} \mathrm{CO}$ data. The ${ }^{13} \mathrm{CO}$ column density, $N\left({ }^{13} \mathrm{CO}\right)$, is estimated by

$$
N\left({ }^{13} \mathrm{CO}\right)=2.42 \times 10^{14} \times \frac{\tau\left({ }^{13} \mathrm{CO}\right) T_{\mathrm{ex}}(\mathrm{K}) \Delta V\left(\mathrm{~km} \mathrm{~s}^{-1}\right)}{1-\exp \left[-5.29 / T_{\mathrm{ex}}(\mathrm{K})\right]}\left(\mathrm{cm}^{-2}\right) .
$$

The ratio of $N\left(\mathrm{H}_{2}\right) / N\left({ }^{13} \mathrm{CO}\right)$ was assumed to be $7 \times 10^{5}$ (Dickman 1978). The $M_{\mathrm{LTE}}$ of a cloud from $N\left(\mathrm{H}_{2}\right)$ is derived by the same way as ${ }^{12} \mathrm{CO}$ (see equation (1)). The column density and $M_{\mathrm{LTE}}$ range from $2.3 \times 10^{20}$ to $1.7 \times 10^{21} \mathrm{~cm}^{-2}$ and 0.03 to $1.41 M_{\odot}$, respectively, where the detection limit in the column density is $2.0 \times 10^{20} \mathrm{~cm}^{-2}$, coressponding to mass limit of $0.009 M_{\odot}$, smaller than that of ${ }^{12} \mathrm{CO}$ because the observations of ${ }^{13} \mathrm{CO}$ were made by higher grid sampling and lower rms noise fluctuations than those of ${ }^{12} \mathrm{CO}$, respectively. Figure 6 shows the histograms of each physical property. The virial mass, $M_{\text {vir }}$, of a cloud was derived by using the following equation, assuming isothermal, spherical, and uniform density distribution with no external magnetic pressure:

$$
M_{\mathrm{vir}}=209 \times R \times \Delta V_{\mathrm{comp}}^{2},
$$

where $R$ and $\Delta V_{\text {comp }}$ are the radius (pc) and line width $\left(\mathrm{km} \mathrm{s}^{-1}\right)$ of the composite profile obtained by averaging all the spectra within a cloud, respectively (for details of the line width of composite profiles, see Yonekura et al. 1997; Kawamura et al. 1998). From this equation, $M_{\text {vir }}$ is estimated to be in a range from 4.7 to $197 M_{\odot}$. These physical properties are also listed in Table 2 .

\section{CORRELATIONS AMONG THE CLOUD PHYSICAL PARAMETERS}

\subsection{Mass Spectrum and Size Linewdith Relation}

Figure $7 \mathrm{a}$ and $7 \mathrm{~b}$ show the mass spectrum of the present ${ }^{12} \mathrm{CO}$ and ${ }^{13} \mathrm{CO}$ clouds. The spectra have been fitted by the maximum-likelihood method (Crawford et al. 1970), and it is found that they are well fitted by a single power law as follows; $d N / d M \propto M^{-1.53 \pm 0.13}$ for 
the ${ }^{12} \mathrm{CO}$ clouds and $d N / d M \propto M^{-1.36 \pm 0.10}$ for the ${ }^{13} \mathrm{CO}$ clouds. These values of the spectral indices seem to be similar to those for the higher mass range (e.g., Yonekura et al. 1997).

Figure 8 shows a plot of size, $R$, versus line width, $\Delta V$, of the ${ }^{13} \mathrm{CO}$ clouds in this region and for a comparison with other HLCs, MBM 53, 54, and 55 complex (Yamamoto et al. 2003). We can make fitting as follows by using a least-squares fitting, $\log (\Delta V)$ $=(0.22 \pm 0.43) \times \log (R)+(0.37 \pm 0.52)($ c.c. $=0.23)$ for the present region and $\log (\Delta V)=$ $(0.43 \pm 0.32) \times \log (R)+(0.53 \pm 0.28)($ c.c. $=0.37)$ for MBM 53, 54, and 55 complex. The low correlation coefficient (c.c.) indicates that there is no significant correlation between $R$ and $\Delta V$ because of a small range of $R$. Here we do not show the same relationship for ${ }^{12} \mathrm{CO}$, because the non-circular shape of the ${ }^{12} \mathrm{CO}$ clouds may not be appropriate to derive reliable $R$.

\section{2. $M_{\text {LTE }}$ vs. $M_{\text {vir }}$}

Figure 9 shows a plot of $M_{\mathrm{LTE}}$ versus $M_{\mathrm{vir}}$. The present ${ }^{13} \mathrm{CO}$ clouds are located far above the equilibrium line where $M_{\mathrm{LTE}}$ is equal to $M_{\mathrm{vir}}$, indicating that the ${ }^{13} \mathrm{CO}$ clouds are not in the virial equilibrium. This indicates that none of the molecular clouds are gravitationally bound. These parameters can be fitted by using a least-squares fitting as follows, $\log \left(M_{\mathrm{vir}}\right)$ $=(0.91 \pm 0.30) \times \log \left(M_{\mathrm{LTE}}\right)+(2.23 \pm 0.29) \quad($ c.c. $=0.66)$ for present molecular clouds and $\log \left(M_{\mathrm{vir}}\right)=(0.77 \pm 0.13) \times \log \left(M_{\mathrm{LTE}}\right)+(0.16 \pm 0.08)($ c.c. $=0.74)$ for MBM53, 54 , and 55 complex. As mentioned in Yamamoto et al. (2003), the present molecular clouds also tend to be more virialized as the mass increases. For the Gemini and Auriga, and CepheusCassiopeia regions, the indices of $M_{\mathrm{LTE}}$ for $M_{\text {vir }}$ of ${ }^{13} \mathrm{CO}$ clouds are estimated to be $0.72 \pm 0.03$ and $0.62 \pm 0.03$ for the cloud mass range of $M_{\mathrm{LTE}}<10^{4} M_{\odot}$ and $10^{2} M_{\odot}<M_{\mathrm{LTE}}<10^{5} M_{\odot}$, respectively (Kawamura et al. 1998; Yonekura et al. 1997). Although the mass ranges of MBM 53, 54, and 55 complex and of this region are $10^{-1} M_{\odot}<M_{\mathrm{LTE}}<10^{2} M_{\odot}$ and $10^{-2}$ $M_{\odot}<M_{\mathrm{LTE}}<1 M_{\odot}$, respectively, difference in the power-law indices among these regions is small and a tendency that the SCs have large ratios of $M_{\mathrm{vir}} / M_{\mathrm{LTE}}$ is commonly seen.

\section{COMPARISON WITH OTHER WAVELENGTH DATA}

\subsection{No Sign of Star Formation}

In order to look for sings of star formation associated with the present molecular clouds, we searched the IRAS point source catalog for candidates of protostellar objects satisfying the following criteria: (1) point sources having a data quality flag better than 2 in 4 bands, 
(2) flux ratios at $12,25,60 \mu \mathrm{m}$ satisfying both $\log \left(F_{12} / F_{25}\right)<-0.3$ and $\log \left(F_{25} / F_{60}\right)<0$, and not identified as galaxies or planetary nebulae and stars. We find that there are no cold IRAS point sources satisfying these criteria in the present molecular clouds. We also find that there are no IRAS point sources having a spectrum like a T-Tauri type star or no YSOs identified from Point Source Catalog of Two-Micron All-Sky Survey in this region. Here we select the 2MASS sources whose signal to noise ratio of valid measurements in all bands are greater than 10 and extract the sources which have the spectra like T Tauri stars in $(J-H)-(H-K)$ color-color diagram (e.g., Meyer et al. 1997) . These results suggest that the present molecular clouds are not the site of recent star-formation, or that the region is

not remnants of past star formation. Such a low level of star formation is similar to the other HLCs including MBM 53, 54, and 55 complexes.

\subsection{Comparison with HI}

Figure 10 shows the integrated intensity map of H I taken from a Leiden-Dwingeloo H I survey (Hartmann \& Burton 1997) superposed on the integrated intensity of CO. The integrated velocity range is from -16 to $0 \mathrm{~km} \mathrm{~s}^{-1}$, corresponding to the velocity range of the ${ }^{12} \mathrm{CO}$ emission. Because an angular resolution of $\sim 30^{\prime}$ is coarser than that of the present CO observations by a factor of $\sim 10$, we discuss here only the overall comparison between $\mathrm{CO}$ and HI distributions. The HI distribution is loop-like and the molecular clouds are distributed nearly along the H I loop.

Figure 11 shows the position-velocity diagram of H I integrated from -40.5 to -39.5 and -52.5 to -51.5 in Galactic latitude, respectively. The hole like structures can be seen in HI, suggesting that these H I clouds are expanding. These expanding structures in Figures 11(a) and 11(b) correspond to the Galactic northern and southern HI shells shown as the thick dashed (semi) ellipses in Figure 10, respectively, while the expanding motion is not seen in CO (See Figure 3). These two expanding shells are also identified by an infrared radiation (Kiss et al. 2004). From Figure 10, an H I cloud around $(l, b) \sim\left(109^{\circ},-52^{\circ}\right)$ seems to be located on the left side of the southern expanding shell and the molecular clouds are distributed nearby. It is difficult to distinguish with which H I shell the molecular clouds located around $(l, b) \sim\left(109^{\circ},-52^{\circ}\right)$ are associated because the HI velocities of the two shells are similar with each other. The shape of ${ }^{12} \mathrm{CO}$ and SFD100 $\mu \mathrm{m}$ radiation around $(l, b) \sim\left(109^{\circ},-52^{\circ}\right)$ in Figure 2 is also similar to the left side of the expanding shell. If this is true, there may be two expanding structures in the present region. HD886 (109.43, $-46^{\circ} .68$ ) is located near the center of the northern expanding shell, indicating that HD886 may be affecting the northern expanding shell. The parallax of HD886 has been measured 
to be $9.79 \pm 0.81$ mas (Perryman et al. 1997), corresponding to a distance from the Sun of $102_{-8}^{+9}$ pc. The proper motion has also been measured to be $\mu \alpha *=0^{\prime \prime} 0047 \mathrm{yr}^{-1}, \mu \delta=-0^{\prime} \cdot 0082$ $\mathrm{yr}^{-1}$ by Perryman et al. (1997). From this proper motion, the velocity of HD886 in the L-B map is estimated to be $\sim 4.3 \times 10^{-6} \mathrm{pc} \mathrm{yr}^{-1}$ at $\sim 100 \mathrm{pc}$ (see Figure 10). We use typical values of the stellar wind for B2(IV) star on $d M / d t=10^{-9} M_{\odot} \mathrm{yr}^{-1}$ and $V_{\infty}=1000 \mathrm{~km}$ $\mathrm{s}^{-1}$ (e.g., Snow 1982). From these parameters, the energy injected to the northern shell is estimated to be $\sim 10^{47}$ ergs in a few $\times 10^{6}$ yr. The expanding energy of the northern shell is estimated to be $\sim 10^{48}$ ergs from the atomic and molecular hydrogen, using that the masses of amomic and molecular hydrogen associated with the northern shell are $\sim 400$ and $42 M_{\odot}$, respectively, the expanding velocity is $\sim 7 \mathrm{~km} \mathrm{~s}^{-1}$ which is estimated from Figure 11 and the equation of $E_{\exp }=1 / 2 M V_{\exp }^{2}$. Since the expanding energy of the atomic and molecular hydrogen is comparable to the energy from HD886, additional source of energy other than HD886 such as photo evaporation is needed to explain the expanding energy because the energy conversion efficiency of the stellar wind is $\lesssim 10 \%$. The energy of the southern shell is

estimated to be $\sim 10^{47} \mathrm{ergs}$, by using that the masses of atomic and molecular hydrogen are $\sim 1000$ and $16 M_{\odot}$, respectively, and that the expanding velocity is $\sim 9 \mathrm{~km} \mathrm{~s}^{-1}$. We could not find possible candidates of the energy source for the expanding feature in the literature (SIMBAD) and there are no counterparts in optical or X-ray wavelength. Although we could not identify the possible candidates, we cannot exclude a possibility that these objects may have escaped from the region in a few $\times 10^{6-7} \mathrm{yr}$ after forming these structures.

\section{DISCUSSION}

\subsection{Physical States of the Small Clouds}

The present observations have revealed numerous molecular clouds having very small mass of less than $1 M_{\odot}$. It is of considerable interest to pursue the physical states of these SCs from view points of cloud physics and chemistry as well as of the origin of molecular clouds.

We shall hereafter focus on the low mass ${ }^{12} \mathrm{CO}$ clouds whose mass is less than $1 M_{\odot}$. The total number of such clouds is 65 among 78 . The ${ }^{13} \mathrm{CO}$ emission has been searched for toward 24 of the $43{ }^{12} \mathrm{CO}$ low-mass clouds whose mass is in a range of $0.1-1.0 M_{\odot}$ and has been detected from 8 of them. Figure 12 shows correlations of the molecular column density, estimated from ${ }^{12} \mathrm{CO}$ and ${ }^{13} \mathrm{CO}$, of the clouds where both ${ }^{12} \mathrm{CO}$ and ${ }^{13} \mathrm{CO}$ emission are detected. It is seen that almost all of the ${ }^{12} \mathrm{CO}$ clouds having molecular column density greater than $5 \times 10^{20} \mathrm{~cm}^{-2}$, corresponding to the visual extinction of 0.55 mag if we use the relationship of $N\left(\mathrm{H}_{2}\right)=9.4 \times 10^{20} \times A \mathrm{v} \mathrm{cm}^{-2}$ (Bohlin et al. 1978; Hayakawa et al. 1999), show 
significant ${ }^{13} \mathrm{CO}$ emission. We note that there are $22{ }^{12} \mathrm{CO}$ clouds whose mass is less than $0.1 M_{\odot}$; for those it is doubtful that the ${ }^{13} \mathrm{CO}$ emission is so significant as those whose mass is a range of $0.1-1.0 M_{\odot}$ although only 3 of them were searched for the ${ }^{13} \mathrm{CO}$ emission in the present study. We note that we ignore the possible contribution of atomic hydrogen in the above relationship. If we take into account the distribution of atomic hydrogen as $N(\mathrm{HI})$, the visual extinction would increase by $0.2 \sim 0.3 \mathrm{mag}$. This may be explained as that the ${ }^{13} \mathrm{CO}$ emitting regions become significant when $A \mathrm{v}$ becomes larger than $\sim 1 \mathrm{mag}$, marginally enough to shield the ultraviolet radiation to protect ${ }^{13} \mathrm{CO}$ molecules (e.g., Warin et al. 1996), although ${ }^{13} \mathrm{CO}$ molecules may be also affected from the ultraviolet radiation because the $N\left(\mathrm{H}_{2}\right)$ derived from ${ }^{13} \mathrm{CO}$ is lower than that derived from ${ }^{12} \mathrm{CO}$ in most of molecular clouds.

The peak intensity ratio of ${ }^{12} \mathrm{CO}$ and ${ }^{13} \mathrm{CO}$ is around 5 , much smaller than the terrestrial abundance ratio of 89 , indicating that the ${ }^{12} \mathrm{CO}$ emission is optically thick in the clouds where the both emissions are detected. The maximum ${ }^{12} \mathrm{CO}$ peak temperature of the present brightest ${ }^{12} \mathrm{CO}$ emission is $6 \mathrm{~K}$, and this suggests that the excitation temperature is consistent with the kinetic temperature of $\sim 10 \mathrm{~K}$, typical to the local dark clouds. The low mass clouds show lower ${ }^{12} \mathrm{CO}$ peak temperatures down to $1 \mathrm{~K}$, significantly less than the brightest peak intensities. It is not clear if this is due to the lower excitation temperatures or due to smaller filling factors significantly less than 1 . In order to clarify this point we need observations of the present low mass clouds at much higher angular resolutions.

\subsection{Origin of the Very Small Clouds}

Figure 13 shows the histograms of mass and sizes of ${ }^{12} \mathrm{CO}$ clouds. In the present region, we have detected a large number of molecular clouds of mass less than $0.1 M_{\odot}$ and sizes less than $0.1 \mathrm{pc}$ not detected so far in the other regions. We may ask why molecular clouds of mass less than $0.1 M_{\odot}$ and size less than $0.1 \mathrm{pc}$ such as the present clouds have not been detected so far. The main reason for this is perhaps the paucity of high-resolution observational studies of nearby molecular clouds. Most of the observations of the local high latitude clouds were made at lower resolutions of 10 arc-min or at a coarse grid spacing of 1 degree, both of which are unable to detect and resolve the present low mass clouds. This suggests that the low mass clouds similar to the present ones may not be uncommon in the interstellar space and warrant more extensive searches for them in the other parts of the sky.

It is interesting to compare the physical parameters of the present VSCs with theoretical studies. The typical size of these HLCs, $\sim 0.1 \mathrm{pc}$, is significantly less than the Jeans length of $1.3 \mathrm{pc}-7 \mathrm{pc}$ for molecular gas with $T=10 \mathrm{~K}$ and $n\left(\mathrm{H}_{2}\right)=10-100 \mathrm{~cm}^{-3}$. If we take temperature 
higher than $10 \mathrm{~K}$, the length becomes even larger. Figure 14 shows the radial distribution of mass surface density which is derived by dividing the mass in circular annulus of a radius by the area of the circular annulus. Mass surface density in the present region is fairly flat in radius because there is no massive molecular cloud in the center of the VSCs. On the other hand, mass surface density in the other regions has a gradient for radius, indicating that there are small molecular clouds around a massive molecular cloud whose mass is several dozen $M_{\odot}$ or greater (e.g., Sakamoto 2002, Sakamoto \& Sunada 2003).

In these regions, the gravity of the massive molecular cloud may contribure to the formation of these small clouds by increasing the pressure in the surroundings. These suggest that mechanisms other than gravitational instability might contribute to the formation of present VSCs. A theory of molcular cloud formation is discussed by Koyama \& Inutsuka (2002). According to them, molecular clouds smaller than the Jeans-length can be formed in the shocked layer through the thermal instability. We shall present some considerations by comparing the observational results with their theoretical results.

Present VSCs are likely to be affected by HD886 in the last few $\times 10^{6}$ yr (for details, see section 5.2). Although the mechanical luminosity from HD886 injected to the loop-like structure during a few $\times 10^{6} \mathrm{yr}$ is low to explain the expanding of the interstellar matter, it is a possibility that the stellar wind of HD886 is the source of shock. Koyama \& Inutsuka (2002) assumed shock velocity of $26 \mathrm{~km} \mathrm{~s}^{-1}$ and density of $0.6 \mathrm{~cm}^{-3}$ as an initial pre-shock condition and find that the region of density greater than $100 \mathrm{~cm}^{-3}$ grows in size to $\sim 0.2 \mathrm{pc}$ $\times 0.1 \mathrm{pc}$ in $1.06 \times 10^{6} \mathrm{yr}$ and the internal structure consists of some filaments. The velocity dispersion of CO derived by Koyama \& Inutsuka (2002) is a few $\mathrm{km} \mathrm{s}^{-1}$. The size of the smallest molecular clouds and the velocity dispersion of $\mathrm{CO}$ are comparable to those derived from Koyama \& Inutsuka (2002). We cannot resolve the internal structure of the VSCs because the present VSCs are detected with only a few points for each. The typical column density derived by Koyama \& Inutsuka (2002) is $2 \times 10^{20} \mathrm{~cm}^{-2}$, while the column density of the VSCs is estimated to be $\sim 1.6 \times 10^{20} \mathrm{~cm}^{-2}$ (see Figure 4). The surface filling factor of the region of density greater than $100 \mathrm{~cm}^{-3}$ in Koyama \& Inutsuka (2002) is roughly estimated to be $30-40 \%$. In this surface filling factor the column density estimated from the observations is consistent with that derived from Koyama \& Inutsuka (2002) and the low temperature of the VSCs is consistent with the result that their peak temperature is lower than that of the typical local dark clouds. These results indicate a potential that the present VSCs are formed in the shock compressed layer through thermal instability. In order to compare the observational results with the theoretical simulation on internal temperature and density structure in more details, the observations of higher resolutions are needed. 


\section{CONCLUSIONS}

We have made a large-scale survey of high Galactic latitude molecular clouds in the $J=1-0$ lines of ${ }^{12} \mathrm{CO}$ and ${ }^{13} \mathrm{CO}$ toward a large scale structure located around $(l, b) \sim$ $\left(109^{\circ},-45^{\circ}\right)$ with NANTEN. This survey spatially resolved the distribution of molecular gas associated with the large scale structure. The main conclusions of the present study are summarized as follows:

1. The ${ }^{12} \mathrm{CO}$ observation covered the entire large loop-like structure. The loop-like structure consits of very small clumpy clouds. The ${ }^{12} \mathrm{CO}$ clouds are concentrated on the north to north-west of the loop-like structure and toward the south of that. We identified $78{ }^{12} \mathrm{CO}$ clouds in the observed region. The total mass is estimated to be $\sim 64 M_{\odot}$ if we assume the conversion factor from $\mathrm{CO}$ intensity to $N\left(\mathrm{H}_{2}\right)$ as $1.0 \times 10^{20} \mathrm{~cm}^{-2} /(\mathrm{K}$ $\left.\mathrm{km} \mathrm{s}^{-1}\right)$.

2. We performed ${ }^{13} \mathrm{CO}$ observations in and around the whole area where the peak temperature of ${ }^{12} \mathrm{CO}$ is more than $2.0 \mathrm{~K}$. We identified $33{ }^{13} \mathrm{CO}$ clouds and derived physical properties under the assumption of LTE.

3. The mass spectra are well fitted by a power law, $d N / d M \propto M^{-1.53 \pm 0.13}$ for the ${ }^{12} \mathrm{CO}$ clouds and $d N / d M \propto M^{-1.36 \pm 0.10}$ for the ${ }^{13} \mathrm{CO}$ clouds. These spectral indices are similar to those derived in the other regions.

4. The size and the line width relation of ${ }^{13} \mathrm{CO}$ clouds is fitted by a least-squares method, $\log (\Delta V)=(0.22 \pm 0.43) \times \log (R)+(0.37 \pm 0.52)$ (c.c. $=0.23)$, but the correlation is not good.

5. Present ${ }^{13} \mathrm{CO}$ clouds are far from the virial equilibrium, indicating that ${ }^{13} \mathrm{CO}$ clouds are not gravitationally bound. $M_{\text {vir }}$ and $M_{\text {LTE }}$ relation can be fitted by a least-squares method as $\log \left(M_{\text {vir }}\right)=(0.91 \pm 0.30) \times \log \left(M_{\mathrm{LTE}}\right)+(2.23 \pm 0.29)($ c.c. $=0.66)$. This index is slightly different from the indices in the other regions although the tendency that molecular clouds are more vilialized as the mass increases is consistent with the other regions.

6. There is no sign of star formation from the comparison of IRAS point sources and Point Source Catalog of Two-Micron All-Sky survey in the present region. This suggests that molecular clouds in this region are not the site of present star formation or the remnants of past star formation.

7. There may be two expanding shells in the present region as inferred from H I although we cannot identify them from CO. The total mechanical luminosity of HD886 during 
the last few $\times 10^{6} \mathrm{yr}$ is comparable to the expanding energy of the northern expanding H I shell. This indicates that some additional source of energy other than HD886 is needed to explain the expanding energy.

8. ${ }^{13} \mathrm{CO}$ emission is significantly detected in the ${ }^{12} \mathrm{CO}$ clouds having molecular column density greater than $5 \times 10^{20} \mathrm{~cm}^{-2}$. This may be explained as that the ${ }^{13} \mathrm{CO}$ emitting regions become significant when $A \mathrm{v}$ becomes larger than $\sim 1 \mathrm{mag}$, marginally enough to shield the ultraviolet radiation to protect ${ }^{13} \mathrm{CO}$ molecules.

9. There is a possibility that very small clouds have been formed in the shoked layer through the thermal instability. The stellar wind of HD886 may be the source to creat shocks, forming the loop-like structure where the very small clouds are embedded.

We greatly appreciate the hospitality of all staff members of the Las Campanas Observatory of the Carnegie Institution of Washington. The NANTEN telescope is operated based on a mutual agreement between Nagoya University and the Carnegie Institution of Washington. We also acknowledge that the operation of NANTEN can be realized by contributions from many Japanese public donators and companies. Three of the authors (N.M., T.O., and Y.F.) acknowledge financial support from the scientist exchange program under bilateral agreement between JSPS (Japan Society for the Promotion of Science) and CONICYT (the Chilean National Commission for Scientific and Technological Research). This research has made use of the SIMBAD astronomical database operated by CDS, Strasbourg, France. This publication makes use of data products from the Two Micron All Sky Survey, which is a joint project of the University of Massachusetts and the Infrared Processing and Analysis Center/California Institute of Technology, funded by the National Aeronautics and

Space Administration and the National Science Foundation. This research has made use of the IRAS point sources from the NASA/IPAC Infrared Science Archive, which is operated by the Jet Propulsion Laboratory, California Institute of Technology, under contract with the National Aeronautics and Space Administration.

\section{REFERENCES}

Bhatt, H. C. 2000, A\&A, 362, 715

Blitz, L., Bazell, D., \& Desert, F. X. 1990, ApJ, 352, 13

Bohlin, R. C., Savage, B. D.,\& Drake, J. F. 1978, ApJ, 224, 132 
Crawford, D. F., Jauncey, D. L., \& Murdoch, H. S. 1970, ApJ, 162, 405

Dickman, R. L. 1978, ApJS, 37, 407

Gir, B. Y., Blitz, L., \& Magnani, L. 1994, ApJ, 434, 162

Graedel, T. E., Langer, W. D., \& Frerking, M. A. 1982, ApJS, 48, 321

Hartmann, D., \& Burton, W. B. 1997, Atlas of Galactic Neutral Hydrogen. Cambridge Univ. Press, Cambridge

Hartmann, D., Magnani, L., \& Thaddeus, P., 1998, ApJ, 492, 205

Hayakawa, T., Mizuno, A., Onishi, T., Yonekura, Y., Hara, A., Yamaguchi, R., \& Fukui, Y. 1999, PASJ, 51, 919

Hearty, T., Magnani, L., \& Caillault, J. P. 1999, A\&A, 341, 163

Heithausen, A., \& Thaddeus, P. 1990, ApJ, 353, L49

Kawamura, A., Ohnishi, T., Mizuno, A., Ogawa, H., \& Fukui, Y., 1998, ApJS, 117, 387

Keto, E. R., \& Myers, P. C. 1986, ApJ, 304, 466

Kiss, Cs., Moór, A., Tóth, L. V. 2004 A\&A, 418, 131

Koyama, H., \& Inutsuka, S. 2002, ApJ, 564, 97

Magnani, L., Blitz, L., \& Mundy, L. 1985, ApJ, 295, 402 (MBM)

Magnani, L., Blitz, L., \& Lada, E. A. 1986, ApJ, 301, 395

Magnani, L., Caillault, J. P., Buchalter, A., \& Beichman, C. A. 1995, ApJS, 96, 159

Magnani, L., Hartmann, D., \& Speck, B, G., 1996, ApJS, 106, 447

Magnani, L., Hartmann, D., Holcomb, S. L., Smith, L. E., \& Thaddeus, P. 2000, ApJ, 535, 167

Meyer, M. R., Calvet, N., \& Hillenbrand, L. A. 1997, AJ, 114, 288

Ogawa, H., Mizuno, A., Hoko, H., \& Fukui, Y. 1990, Int. J. Infrared Millimeter Waves, 11, 717

Onishi, T., Yoshikawa, N., Yamamoto, H., Kawamura, A., Mizuno, A., \& Fukui, Y. 2001, PASJ, 53, 1017 
Perryman, M. A. C., et al. 1997, A\&A, 323, L49(Hipparcos Catalogue)

Pound, M. W. 1996, ApJ, 457, 35

Pound, M. W., \& Goodman, A. A. 1997, ApJ, 482, 334

Reach, W. T., Koo, B. C., \& Heiles, C. 1994, ApJ, 429, 672

Reach, W. T.,Wall, W. F., \& Odegard, N. 1998, ApJ, 507, 507

Sakamoto, S. 2002, ApJ, 565, 1050

Sakamoto, S., \& Sunada, K. 2003, ApJ, 594, 340

Schlegel, D. J., Finkbeiner, D. P., \& Davis, M. 1998, ApJ, 500, 525

Snow, T. P. Jr 1982, ApJ, 253, L39

van Dishoeck, E. F., \& Black, J. H., 1988, ApJ, 334, 771

Warin, S., Benayounm, J. J., \& Viala, Y. P. 1996, A\&A, 308, 535

Yamamoto, H., Onishi, T., Mizuno, A., \& Fukui, Y. 2003, ApJ, 592, 217

Yonekura, Y., Dobashi, K., Mizuno, A., Ogawa, H., \& Fukui, Y., 1997, ApJS, 110, 21

This preprint was prepared with the AAS IATEX macros v5.2. 


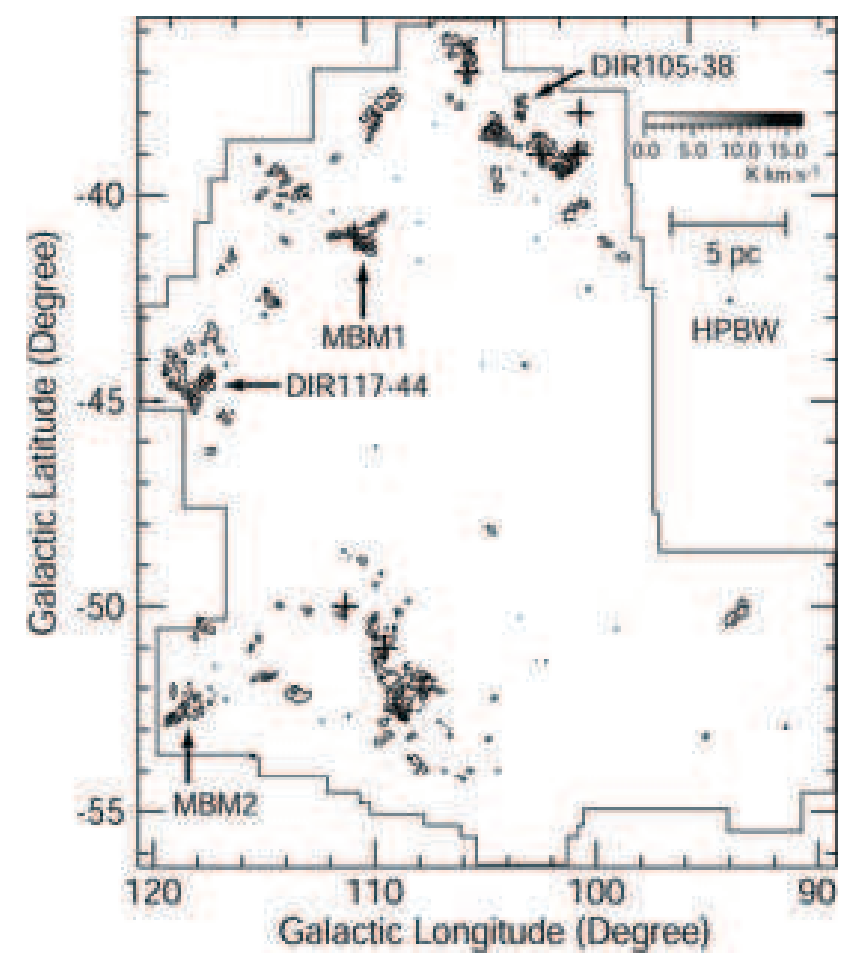

Fig. 1. - Total integrated intensity map of ${ }^{12} \mathrm{CO}(J=1-0)$ shown in Galacticcoordinates. The lowest contour and the separation between contours are 0.77 and $3.08 \mathrm{~K} \mathrm{~km} \mathrm{~s}^{-1}$, respectively. The solid line represents the observed area. The crosses and filled circle indicate the position where Magnani et al. (2000) and (1986) detected ${ }^{12} \mathrm{CO}$, respectively. The locations of DIR clouds in Reach et al. (1998) and MBM clouds in Magnani et al. (1985) are shown in the figure. 


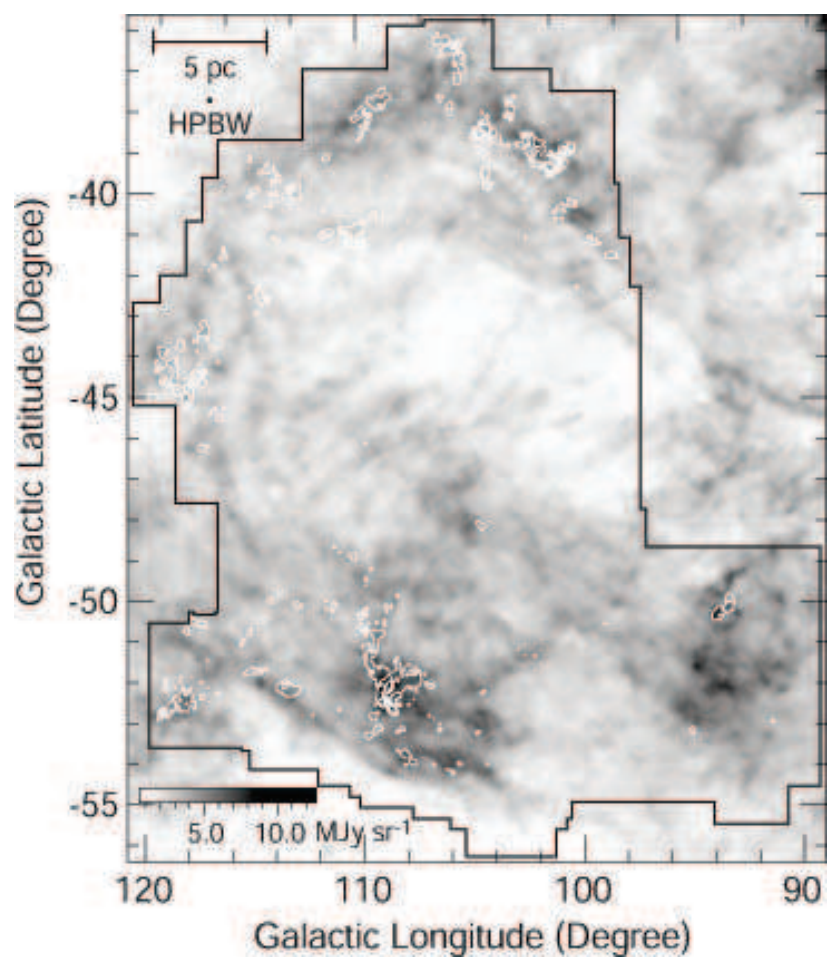

Fig. 2.- Total integrated intensity map of ${ }^{12} \mathrm{CO}(J=1-0)$ superposed on the SFD $100 \mu \mathrm{m}$ image derived by Schlegel et al. (1998). The gray scale shows the intensity of $100 \mu \mathrm{m}$ and white lines represent the integrated intensity of ${ }^{12} \mathrm{CO}$ emission. The lowest contour and the separation between contours are the same as Figure 1. 


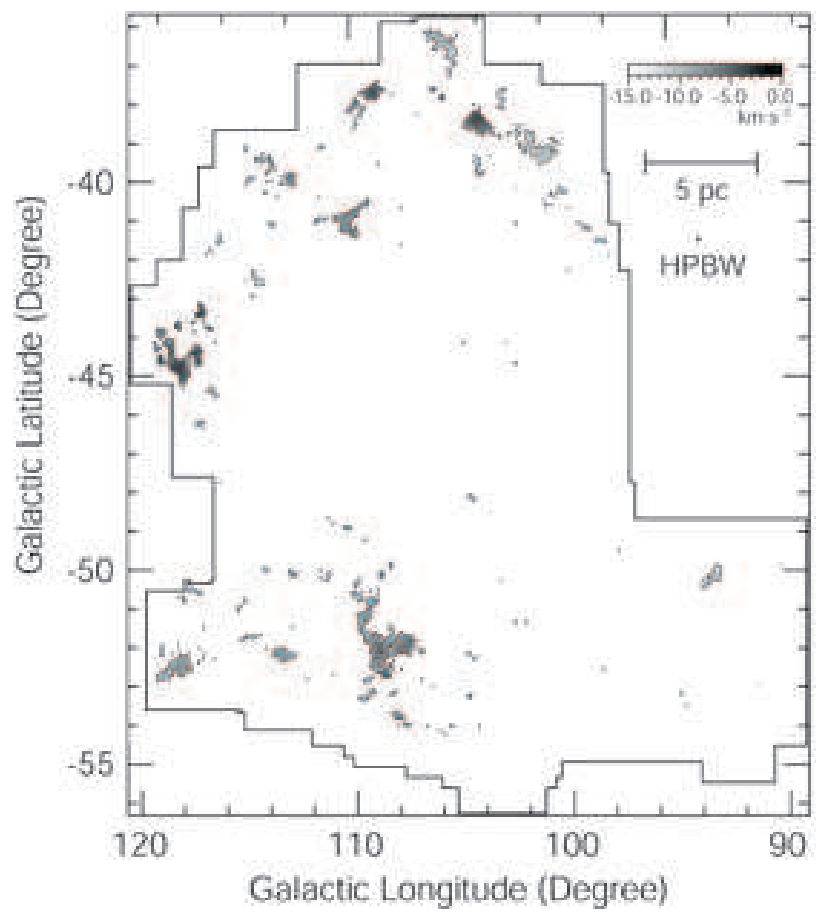

Fig. 3.- The peak radial velocity map of ${ }^{12} \mathrm{CO}(J=1-0)$ emission. The velocity of ${ }^{12} \mathrm{CO}$ emission is derived from the single gaussian fitting. Solid lines in the observed area show the boundary of the ${ }^{12} \mathrm{CO}$ clouds. 

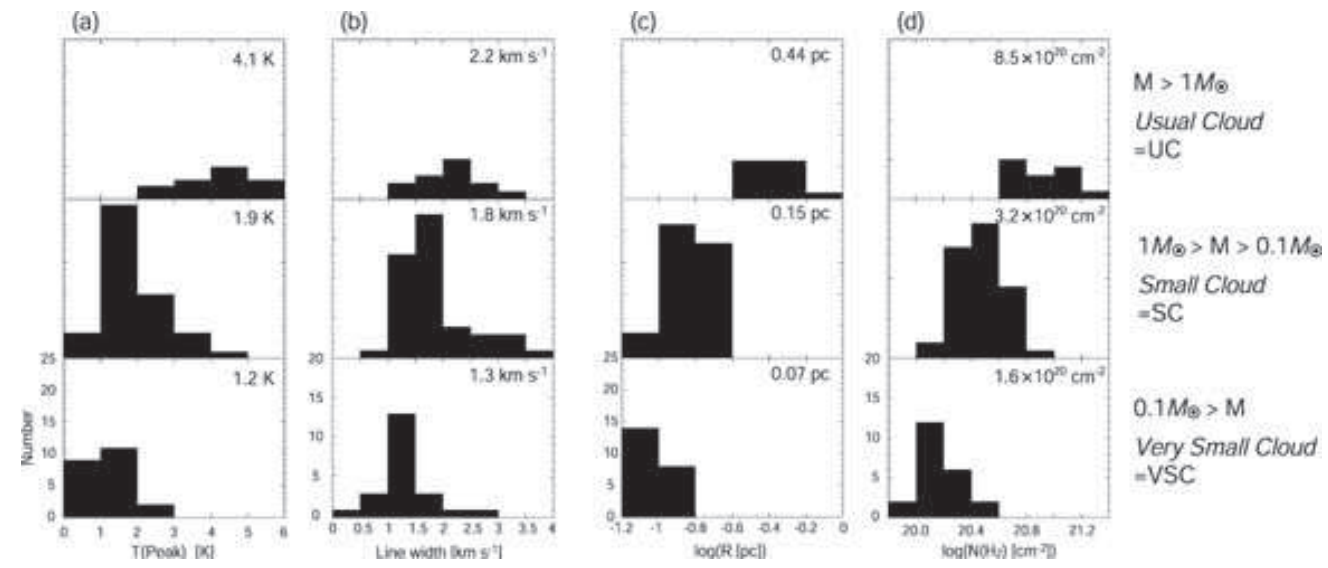

Fig. 4.- Histograms of (a) Peak temperature, (b) Line width, (c) Radius and (d) Column density of molecular hydrogen on ${ }^{12} \mathrm{CO}$ clouds. Histograms of each physical property consist of 3 different ranges on the mass of ${ }^{12} \mathrm{CO}$ clouds. The range of mass of ${ }^{12} \mathrm{CO}$ clouds, $\mathrm{M}$, is $\mathrm{M}>1 M_{\odot}, 1 M_{\odot}>\mathrm{M}>0.1 M_{\odot}$ and $0.1 M_{\odot}>\mathrm{M}$ from the top panel. The number in each panel is the average value. 

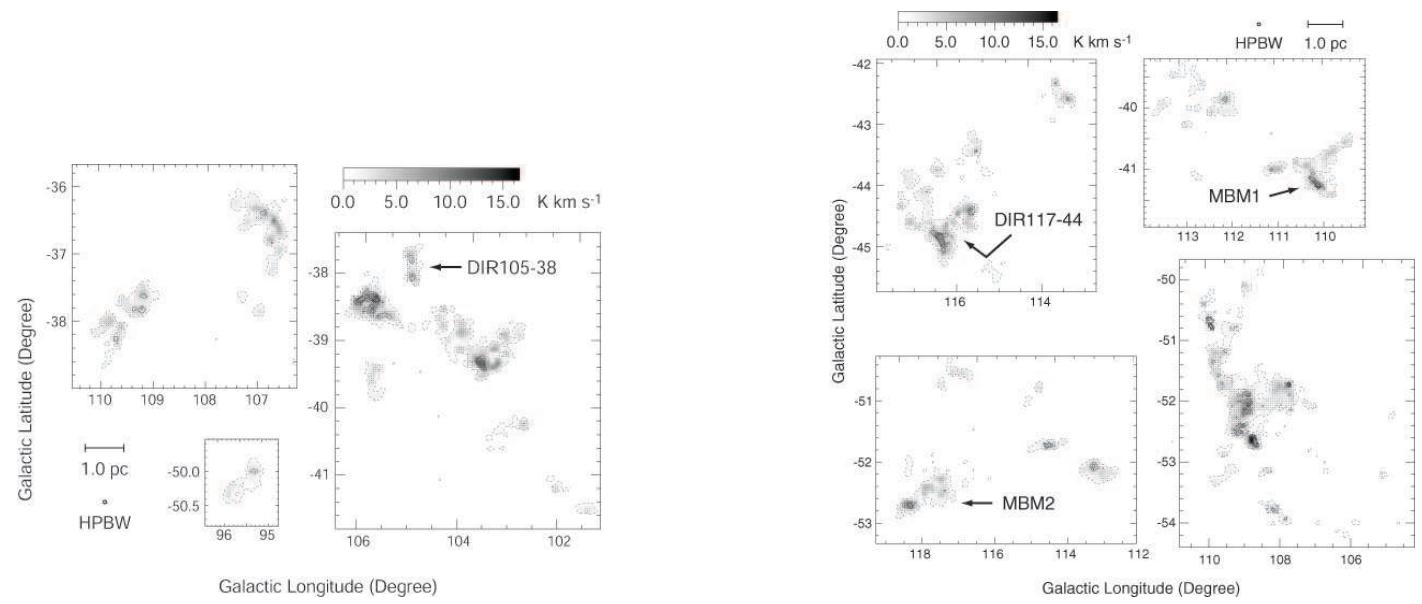

Fig. 5.- Total integrated intensity map of ${ }^{13} \mathrm{CO}(J=1-0)$ shown in Galactic coordinates (bold lines). The lowest contour and the separation between contours are $0.3 \mathrm{~K} \mathrm{~km} \mathrm{~s}^{-1}$. Gray scale shows the velocity integrated intensity map of ${ }^{12} \mathrm{CO}(J=1-0)$. Dashed lines show the boundary of ${ }^{12} \mathrm{CO}$ clouds. Dots indicate the positions observed in ${ }^{13} \mathrm{CO}$. The linear scale of all the figures are the same. 

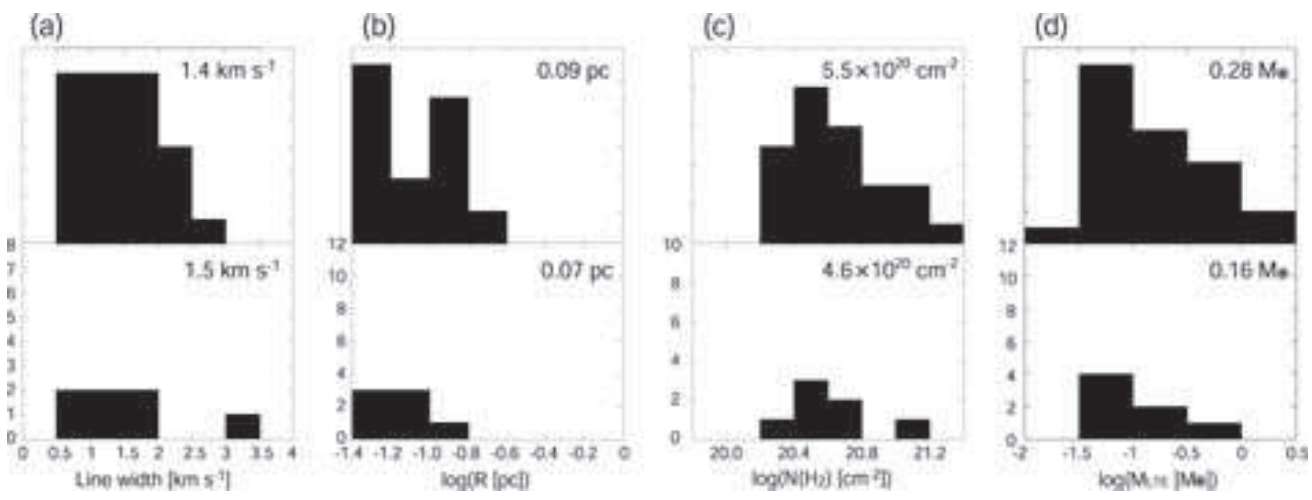

Fig. 6.- Histograms of (a) Line widht, (b) Radius, (c) Column density of molecular hydrogen and (d) LTE mass on the ${ }^{13} \mathrm{CO}$ clouds. The ${ }^{13} \mathrm{CO}$ clouds identified in the UC and SC are shown in the upper and lower histogram, respectively. The range and the interval of the histograms of (a), (b), and (c) are the same as Figure 4. The number in each panel is the average value. 

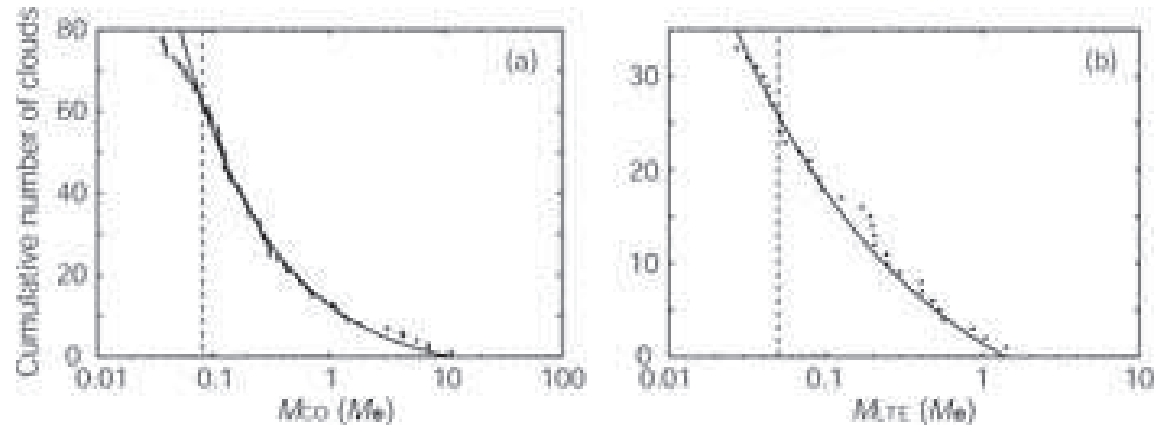

Fig. 7.- (a) Mass spectrum of the ${ }^{12} \mathrm{CO}$ clouds. The number of clouds, $N\left(\geq M_{C O}\right)$, with mass greater than $M_{\mathrm{CO}}$ is plotted against $M_{\mathrm{CO}}$, along with the best-fitting power law (solid lines). The best fitting is expected as $N\left(\geq M_{\mathrm{CO}}\right)=17.33 \times M_{\mathrm{CO}}{ }^{-0.53}-4.78$ for the mass range of $\geq 0.08 M_{\odot}$, which is the completeness limit indicated by the broken line, derived by using the maximum likelihood method (Crawford et al. 1970). (b) Same as (a), but for ${ }^{13} \mathrm{CO}$ clouds with $M_{\mathrm{LTE}}$. The best fitting is expected as $N\left(\geq M_{\mathrm{CO}}\right)=12.54 \times M_{\mathrm{LTE}}{ }^{-0.36}-11.06$ for the mass range of $\geq 0.05 M_{\odot}$. 


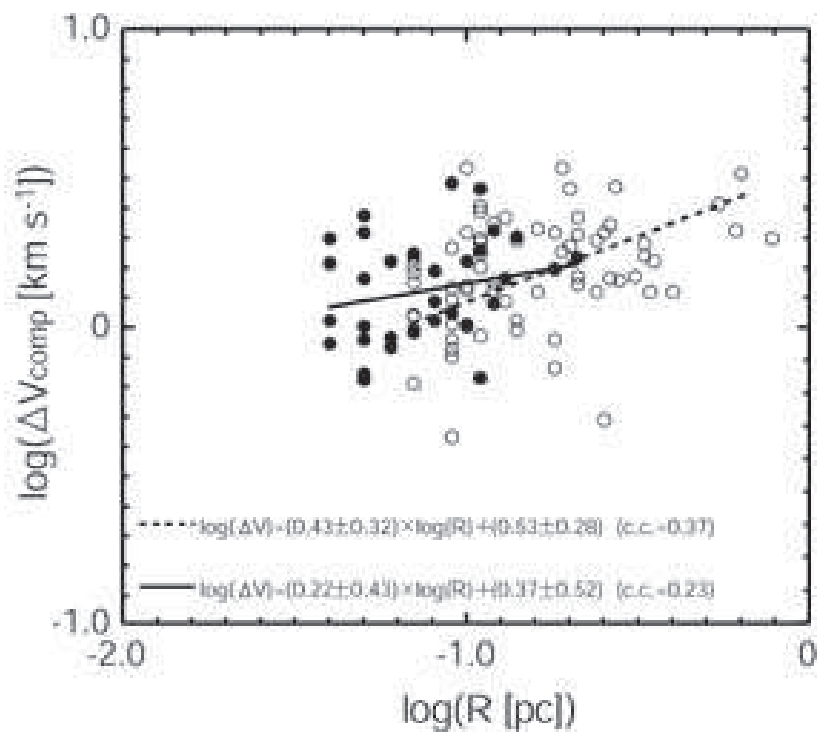

Fig. 8. - Plots of composite line width, $\Delta V_{\text {comp }}$ versus radius of ${ }^{13} \mathrm{CO}$ clouds, $\mathrm{R}$. The filled and open circles indicate the ${ }^{13} \mathrm{CO}$ clouds in present region and those in MBM 53, 54 and 55 complex, respectively. The solid and dashed line indicate the least-squares fit to these plots in present region and MBM 53, 54 and 55, respectively. 


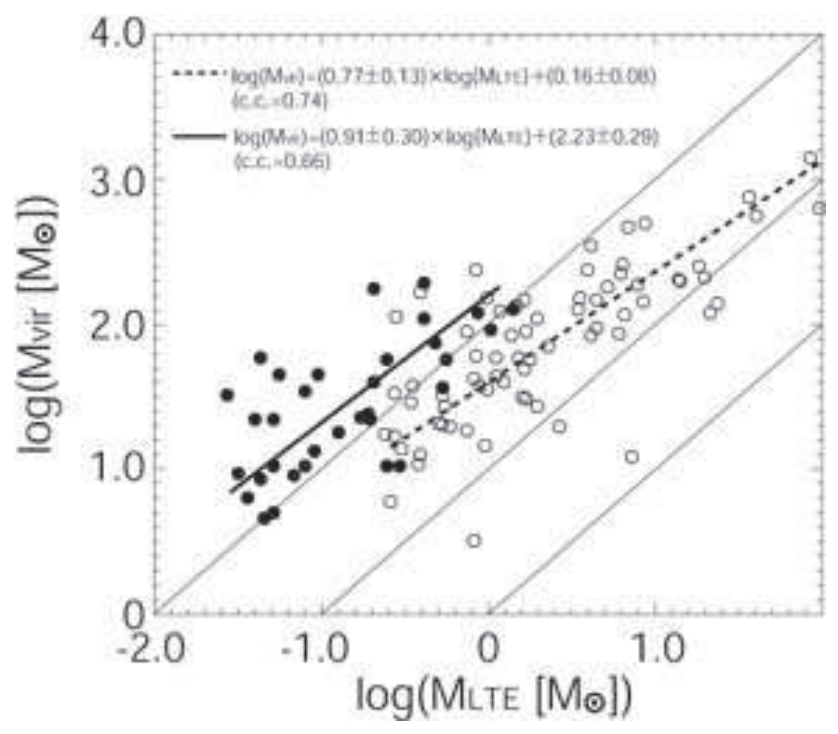

Fig. 9.- Plots of $M_{\text {vir }}$ versus $M_{\mathrm{LTE}}$. The filled and open circles indicate the ${ }^{13} \mathrm{CO}$ cluods in present region and in MBM 53, 54, and 55 complex, respectively. The three thin solid lines represent $M_{\mathrm{vir}}=10^{2} \times M_{\mathrm{LTE}}, M_{\mathrm{vir}}=10 \times M_{\mathrm{LTE}}$ and $M_{\mathrm{vir}}=M_{\mathrm{LTE}}$ from the top. The thick solid and dashed lines represent the least-squares fits to these plots in present region and those MBM 53, 54, and 55 complex, respectively. 


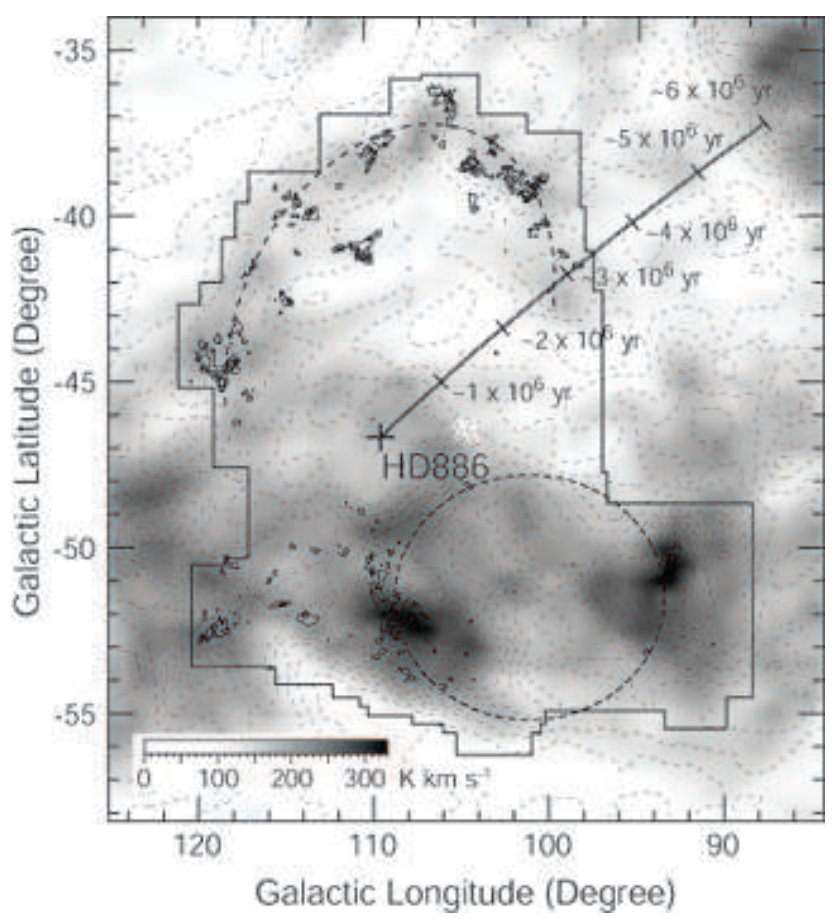

Fig. 10.- Total integrated intensity map of ${ }^{12} \mathrm{CO}(J=1-0)$ superposed on that of H I. The solid contours represent the integrated intensity of ${ }^{12} \mathrm{CO}$. The dashed thin contours and gray scale represent the integrated intensity of $\mathrm{HI}$. The velocity coverage of $\mathrm{HI}$ is -16 to $0 \mathrm{~km}$ $\mathrm{s}^{-1}$, corresponding to that of ${ }^{12} \mathrm{CO}$. The lowest contour and the separation between contours of $\mathrm{HI}$ are $25 \mathrm{~K} \mathrm{~km} \mathrm{~s}^{-1}$ and those of $\mathrm{CO}$ are the same as Figure 1. The thick dashed contours represent the location of the expanding shells. The cross indicates the position of HD886. The path of the proper motion of HD886 is illustrated for the last $\sim 6 \times 10^{6}$ yr by the solid line. 

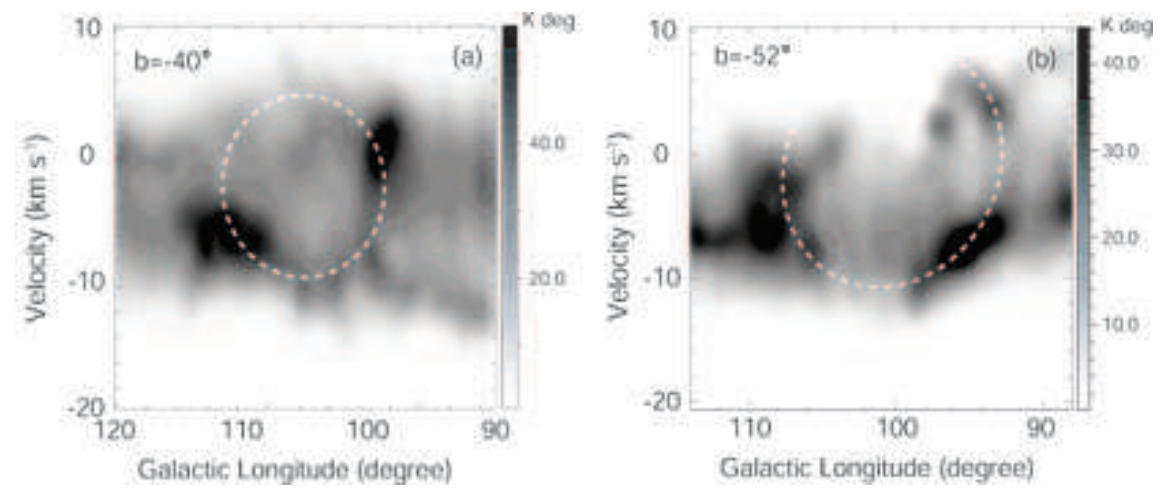

Fig. 11. - Position-velocity map of HI integrated from (a) -40.5 to -39.5 in Galactic latitude, (b) -52.5 to -51.5 in Galactic latitude. The dashed line in each panel represent the location and the extent of the H I holes drawn by hand. 


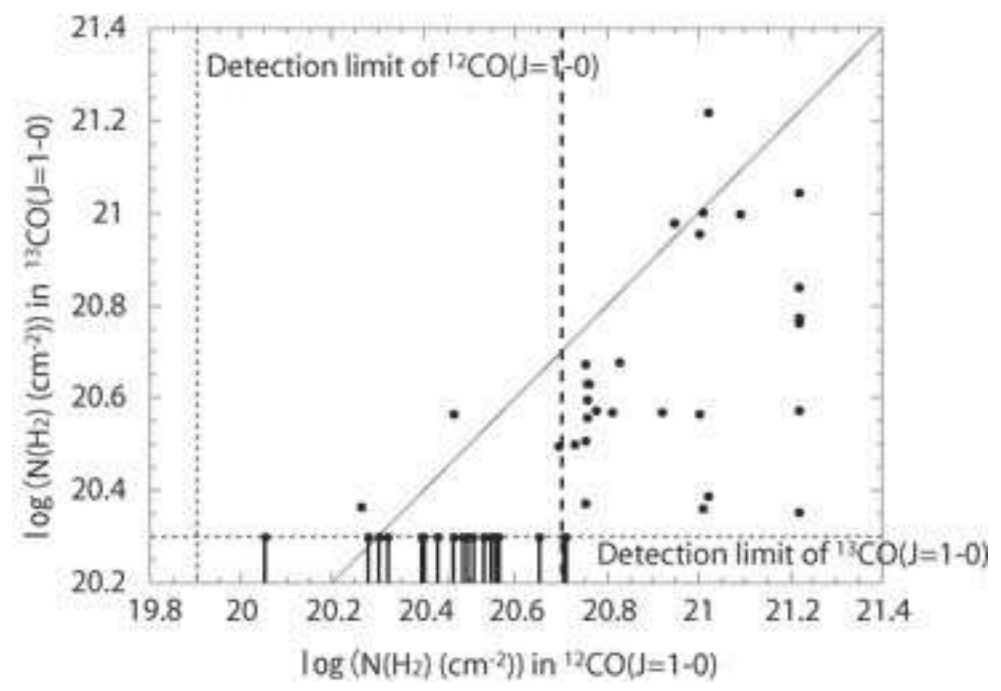

Fig. 12.- Plots of $N\left(\mathrm{H}_{2}\right)$ derived from ${ }^{12} \mathrm{CO}$ versus it from ${ }^{13} \mathrm{CO}$ at the peak position of each cloud which were observed in ${ }^{13} \mathrm{CO}$ emission. The vertical and horizontal dashed lines indicate the detection limit of the observation of ${ }^{12} \mathrm{CO}$ and ${ }^{13} \mathrm{CO}$, respectively. The thick dotted line indicates $N\left(\mathrm{H}_{2}\right)$ from ${ }^{12} \mathrm{CO}=5 \times 10^{20} \mathrm{~cm}^{-2}$ and the solid line indicates the equal one between $N\left(\mathrm{H}_{2}\right)$ derived from ${ }^{12} \mathrm{CO}$ and ${ }^{13} \mathrm{CO}$. 

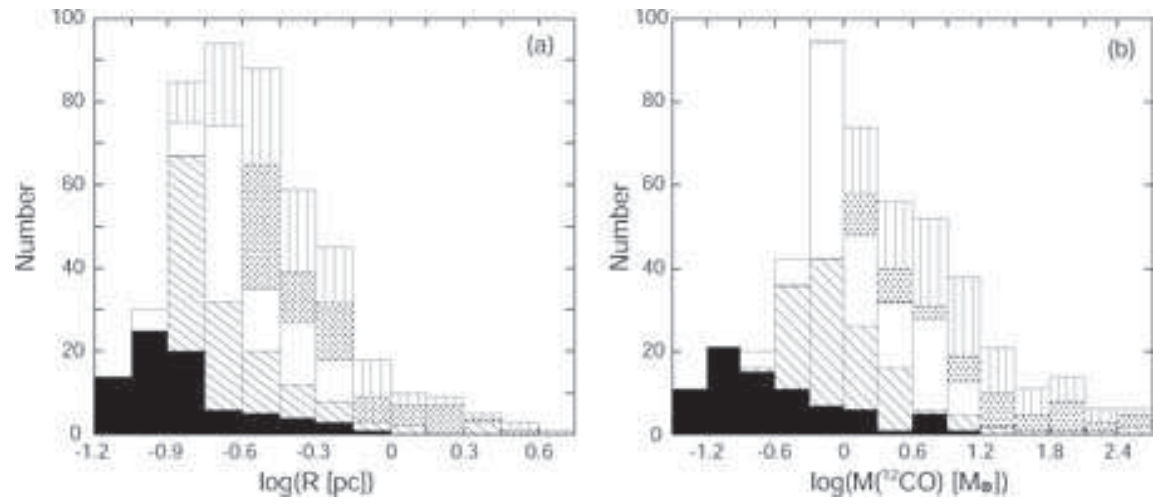

Fig. 13. - Histograms of (a) radii of ${ }^{12} \mathrm{CO}$ clouds and (b) mass of them. The dark shaded areas indicate the ${ }^{12} \mathrm{CO}$ clouds in present region. The vertical lined, the dotted lined, the light shaded and the diagonal lined areas indicate ${ }^{12} \mathrm{CO}$ clouds derived by Tachihara et al. (2001), Magnani et al. (1996), Onishi et al. (2001) and Yamamoto et al. (2003), respectively. 


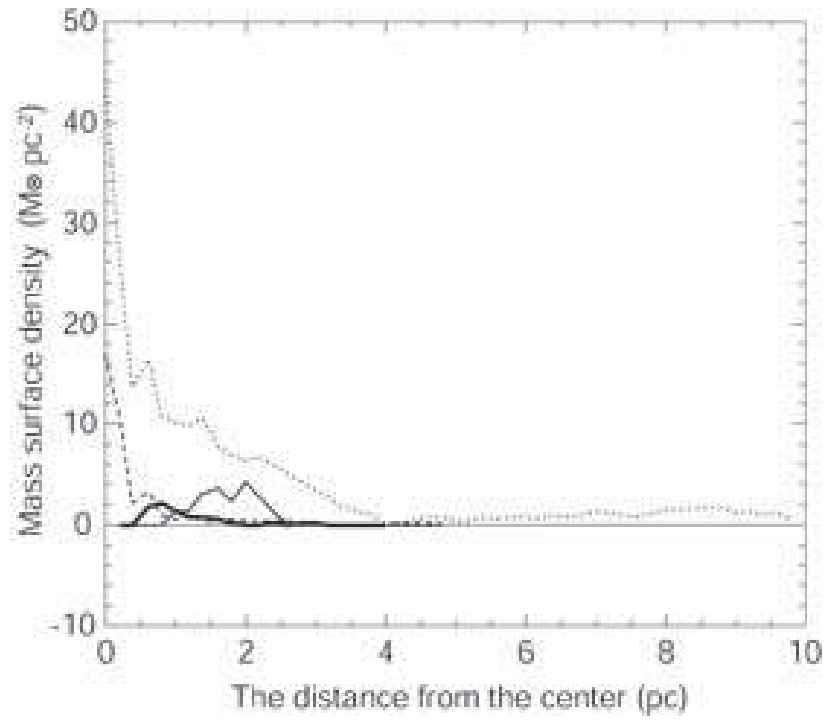

Fig. 14. - The radial distribution of mass surface density. The thick and thin lines indicate the regions around $\left(113^{\circ},-51^{\circ}\right)$ and $\left(113^{\circ},-40^{\circ}\right)$ in Galactic coordinates in present region, respectively. The dashed and dotted lines indicate the regions around HLCG92-35 and MBM 53 (Yamamoto et al. 2003), respectively. 
Table 1. Physical Properties of ${ }^{12} \mathrm{CO}$ Clouds

\begin{tabular}{|c|c|c|c|c|c|c|c|c|}
\hline $\begin{array}{l}\text { No. } \\
\text { (1) }\end{array}$ & $\begin{array}{c}l \\
(2)\end{array}$ & $\begin{array}{c}b \\
(3)\end{array}$ & $\begin{array}{l}T_{\mathrm{R}}^{*} \\
(4)\end{array}$ & $\begin{array}{l}\Delta V \\
(5)\end{array}$ & $\begin{array}{c}V_{\mathrm{LSR}} \\
(6)\end{array}$ & $\begin{array}{c}R \\
(7)\end{array}$ & $\begin{array}{c}N\left(\mathrm{H}_{2}\right) \\
\quad(8)\end{array}$ & $\begin{array}{c}M_{\mathrm{CO}} \\
(9)\end{array}$ \\
\hline 1 & 95.52 & -50.00 & 3.2 & 1.3 & -10.7 & 0.31 & 4.7 & 1.07 \\
\hline 2 & 96.11 & -53.13 & 1.1 & 1.3 & -9.4 & 0.09 & 0.8 & 0.07 \\
\hline 3 & 101.43 & -41.53 & 1.5 & 1.5 & -9.5 & 0.17 & 1.9 & 0.27 \\
\hline 4 & 101.91 & -41.20 & 0.7 & 0.5 & -9.5 & 0.08 & 0.9 & 0.04 \\
\hline 5 & 102.09 & -41.20 & 1.9 & 1.4 & -8.9 & 0.10 & 3.4 & 0.12 \\
\hline 6 & 102.19 & -41.07 & 0.9 & 1.1 & -8.9 & 0.08 & 1.0 & 0.04 \\
\hline 7 & 102.80 & -40.27 & 2.5 & 1.6 & -8.4 & 0.16 & 4.5 & 0.30 \\
\hline 8 & 103.32 & -40.33 & 1.1 & 1.3 & -8.3 & 0.11 & 1.6 & 0.11 \\
\hline 9 & 103.47 & -40.60 & 1.0 & 1.2 & -7.3 & 0.08 & 1.0 & 0.04 \\
\hline 10 & 103.74 & -39.33 & 5.2 & 2.9 & -10.1 & 0.59 & 12.3 & 7.02 \\
\hline 11 & 104.20 & -38.93 & 0.8 & 1.0 & -15.7 & 0.10 & 2.0 & 0.09 \\
\hline 12 & 104.48 & -38.53 & 1.4 & 3.5 & -8.6 & 0.16 & 4.5 & 0.44 \\
\hline 13 & 104.81 & -38.80 & 1.4 & 1.2 & -5.0 & 0.10 & 1.5 & 0.08 \\
\hline 14 & 105.07 & -38.80 & 1.1 & 1.4 & -5.7 & 0.08 & 1.8 & 0.06 \\
\hline 15 & 105.08 & -52.27 & 1.0 & 1.2 & -0.1 & 0.07 & 1.2 & 0.04 \\
\hline 16 & 105.10 & -38.07 & 2.5 & 2.4 & -12.7 & 0.26 & 5.7 & 1.19 \\
\hline 17 & 105.22 & -53.20 & 1.2 & 1.3 & -3.7 & 0.09 & 1.1 & 0.05 \\
\hline 18 & 105.40 & -48.13 & 1.6 & 1.8 & -9.0 & 0.12 & 3.0 & 0.20 \\
\hline 19 & 105.77 & -38.40 & 5.6 & 1.9 & -5.0 & 0.47 & 10.5 & 5.60 \\
\hline 20 & 105.80 & -39.60 & 1.7 & 1.8 & -9.8 & 0.24 & 3.1 & 0.70 \\
\hline 21 & 105.82 & -54.00 & 1.1 & 1.4 & -4.8 & 0.07 & 1.3 & 0.04 \\
\hline 22 & 106.76 & -36.53 & 2.7 & 3.0 & -9.8 & 0.53 & 8.3 & 4.29 \\
\hline 23 & 106.97 & -37.87 & 1.7 & 1.7 & -1.2 & 0.12 & 3.4 & 0.17 \\
\hline 24 & 107.32 & -37.60 & 1.2 & 1.5 & -2.3 & 0.12 & 1.6 & 0.11 \\
\hline 25 & 107.38 & -52.00 & 1.0 & 2.6 & -7.5 & 0.12 & 2.1 & 0.13 \\
\hline 26 & 107.87 & -53.93 & 0.8 & 1.6 & -4.9 & 0.11 & 1.8 & 0.11 \\
\hline 27 & 108.21 & -53.73 & 2.2 & 1.4 & -5.1 & 0.16 & 2.9 & 0.25 \\
\hline 28 & 108.33 & -53.13 & 2.2 & 1.2 & -0.2 & 0.07 & 3.5 & 0.08 \\
\hline 29 & 108.78 & -52.60 & 5.7 & 2.4 & -6.6 & 0.79 & 16.6 & 11.13 \\
\hline 30 & 109.00 & -52.13 & 3.3 & 1.9 & -1.5 & 0.31 & 5.1 & 1.43 \\
\hline
\end{tabular}


Table 1-Continued

\begin{tabular}{|c|c|c|c|c|c|c|c|c|}
\hline $\begin{array}{l}\text { No. } \\
\text { (1) }\end{array}$ & $\begin{array}{c}l \\
(2)\end{array}$ & $\begin{array}{c}b \\
(3)\end{array}$ & $\begin{array}{l}T_{\mathrm{R}}^{*} \\
(4)\end{array}$ & $\begin{array}{l}\Delta V \\
(5)\end{array}$ & $\begin{array}{c}V_{\mathrm{LSR}} \\
(6)\end{array}$ & $\begin{array}{c}R \\
(7)\end{array}$ & $\begin{array}{c}N\left(\mathrm{H}_{2}\right) \\
(8)\end{array}$ & $\begin{array}{c}M_{\mathrm{CO}} \\
(9)\end{array}$ \\
\hline 31 & 109.00 & -50.07 & 2.5 & 1.6 & -4.7 & 0.13 & 5.1 & 0.26 \\
\hline 32 & 109.11 & -50.60 & 0.9 & 2.2 & -5.9 & 0.09 & 1.6 & 0.06 \\
\hline 33 & 109.17 & -37.60 & 4.0 & 1.5 & -4.4 & 0.36 & 5.6 & 1.83 \\
\hline 34 & 109.55 & -52.87 & 1.3 & 2.0 & -7.1 & 0.14 & 2.5 & 0.16 \\
\hline 35 & 109.68 & -38.27 & 4.6 & 1.4 & -8.1 & 0.17 & 5.4 & 0.52 \\
\hline 36 & 109.76 & -38.00 & 2.7 & 2.0 & -4.9 & 0.21 & 4.7 & 0.63 \\
\hline 37 & 109.78 & -53.33 & 2.8 & 1.5 & -6.4 & 0.18 & 2.6 & 0.30 \\
\hline 38 & 109.84 & -50.73 & 4.0 & 2.6 & -7.8 & 0.18 & 5.0 & 0.40 \\
\hline 39 & 109.85 & -38.60 & 0.8 & 1.8 & -8.1 & 0.12 & 2.1 & 0.13 \\
\hline 40 & 110.05 & -50.40 & 2.1 & 3.1 & -7.4 & 0.10 & 3.6 & 0.14 \\
\hline 41 & 110.06 & -41.27 & 4.4 & 2.9 & -6.1 & 0.47 & 10.2 & 4.21 \\
\hline 42 & 110.32 & -48.93 & 2.9 & 0.9 & -4.2 & 0.12 & 2.6 & 0.17 \\
\hline 43 & 110.94 & -41.00 & 1.3 & 3.4 & -6.3 & 0.17 & 5.7 & 0.67 \\
\hline 44 & 110.98 & -39.13 & 1.2 & 2.2 & -9.6 & 0.12 & 2.9 & 0.20 \\
\hline 45 & 111.08 & -50.13 & 1.6 & 1.0 & -7.2 & 0.13 & 1.7 & 0.11 \\
\hline 46 & 112.04 & -39.87 & 3.4 & 2.3 & -5.4 & 0.26 & 6.4 & 1.11 \\
\hline 47 & 112.40 & -40.07 & 1.3 & 2.6 & -7.2 & 0.10 & 3.6 & 0.19 \\
\hline 48 & 112.55 & -39.60 & 1.2 & 1.9 & -3.6 & 0.16 & 3.0 & 0.29 \\
\hline 49 & 112.64 & -50.07 & 0.7 & 1.5 & -6.5 & 0.14 & 1.7 & 0.14 \\
\hline 50 & 112.72 & -41.13 & 1.2 & 1.9 & -6.2 & 0.11 & 2.4 & 0.13 \\
\hline 51 & 112.72 & -41.13 & 1.2 & 1.9 & -6.2 & 0.11 & 2.4 & 0.13 \\
\hline 52 & 112.72 & -39.67 & 1.2 & 2.6 & -5.3 & 0.10 & 1.9 & 0.09 \\
\hline 53 & 112.84 & -40.27 & 1.3 & 1.7 & -5.1 & 0.08 & 2.9 & 0.11 \\
\hline 54 & 113.15 & -39.47 & 1.5 & 1.2 & -4.7 & 0.16 & 2.5 & 0.23 \\
\hline 55 & 113.23 & -52.07 & 4.4 & 1.3 & -7.3 & 0.30 & 5.7 & 1.32 \\
\hline 56 & 113.42 & -42.33 & 3.4 & 2.4 & -10.5 & 0.23 & 5.9 & 0.89 \\
\hline 57 & 113.47 & -39.20 & 0.5 & 1.9 & -5.7 & 0.10 & 1.4 & 0.08 \\
\hline 58 & 113.56 & -49.93 & 1.3 & 0.9 & -7.5 & 0.12 & 1.1 & 0.09 \\
\hline 59 & 113.94 & -51.67 & 1.4 & 1.6 & -9.7 & 0.07 & 3.2 & 0.07 \\
\hline 60 & 114.38 & -51.73 & 3.9 & 1.6 & -9.7 & 0.17 & 6.7 & 0.55 \\
\hline
\end{tabular}


Table 1 - Continued

\begin{tabular}{ccccccccc}
\hline \hline $\begin{array}{c}\text { No. } \\
(1)\end{array}$ & $(2)$ & $(3)$ & $(4)$ & $\begin{array}{c}\Delta V \\
(5)\end{array}$ & $\begin{array}{c}V_{\mathrm{LSR}} \\
(6)\end{array}$ & $\begin{array}{c}R \\
(7)\end{array}$ & $\begin{array}{c}N\left(\mathrm{H}_{2}\right) \\
(8)\end{array}$ & $\begin{array}{c}M_{\mathrm{CO}} \\
(9)\end{array}$ \\
\hline 61 & 114.49 & -50.80 & 1.5 & 1.8 & -7.8 & 0.09 & 3.2 & 0.12 \\
62 & 114.52 & -41.53 & 2.2 & 1.1 & -7.8 & 0.15 & 2.1 & 0.20 \\
63 & 114.83 & -51.07 & 0.8 & 1.8 & -8.1 & 0.10 & 1.4 & 0.07 \\
64 & 114.90 & -41.73 & 1.5 & 1.2 & -8.3 & 0.08 & 1.1 & 0.04 \\
65 & 115.10 & -43.80 & 1.6 & 1.0 & -2.1 & 0.11 & 1.5 & 0.09 \\
66 & 115.16 & -45.33 & 1.2 & 1.6 & -8.4 & 0.17 & 1.6 & 0.25 \\
67 & 115.24 & -43.40 & 1.8 & 2.0 & -3.5 & 0.23 & 2.7 & 0.57 \\
68 & 115.63 & -43.60 & 0.8 & 1.0 & -3.3 & 0.08 & 1.6 & 0.06 \\
69 & 115.74 & -46.20 & 1.5 & 1.0 & -6.8 & 0.13 & 2.3 & 0.18 \\
70 & 116.20 & -43.73 & 3.2 & 1.5 & -2.6 & 0.16 & 3.6 & 0.31 \\
71 & 116.33 & -44.80 & 4.2 & 2.3 & -3.9 & 0.59 & 10.0 & 7.13 \\
72 & 116.45 & -50.53 & 2.3 & 1.1 & -7.3 & 0.17 & 3.5 & 0.35 \\
73 & 116.64 & -52.33 & 0.9 & 1.5 & -7.6 & 0.07 & 2.1 & 0.05 \\
74 & 116.86 & -43.87 & 0.8 & 3.7 & -4.7 & 0.18 & 2.9 & 0.41 \\
75 & 117.01 & -50.73 & 2.7 & 0.8 & -7.5 & 0.10 & 2.0 & 0.10 \\
76 & 117.11 & -44.33 & 2.3 & 1.1 & -2.6 & 0.14 & 5.1 & 0.25 \\
77 & 118.12 & -52.13 & 1.1 & 1.1 & -6.7 & 0.12 & 1.8 & 0.12 \\
78 & 118.23 & -52.67 & 4.2 & 2.0 & -7.8 & 0.43 & 8.8 & 3.12 \\
\hline
\end{tabular}

Note. - Col. (1) : Cloud number, Col. (2)-(3) : Cloud peak $(l, b)$ position in degree, Col. (4) : Peak temperature in K, Col. (5) : Line width of the composite spectrum in $\mathrm{km} \mathrm{s}^{-1}$, Col. (6) : Peak velocity of the composite spectrum in $\mathrm{km} \mathrm{s}^{-1}$, Col. (7) : Radius of the molecular cloud in pc, Col. (8) : Column density of peak position in $10^{20} \mathrm{~cm}^{-2}$, Col. (9) : Mass of the molecular cloud in $M_{\odot}$. Col. (4) to (6) are derived by using a single Gaussiun fitting. 
Table 2. Physical Properties of ${ }^{13} \mathrm{CO}$ Clouds

\begin{tabular}{|c|c|c|c|c|c|c|c|c|c|c|}
\hline $\begin{array}{l}\text { No. } \\
(1)\end{array}$ & $\begin{array}{c}l \\
(2)\end{array}$ & $\begin{array}{c}b \\
(3)\end{array}$ & $\begin{array}{l}T_{\mathrm{R}}^{*} \\
(4)\end{array}$ & $\begin{array}{l}\Delta V \\
(5)\end{array}$ & $\begin{array}{c}V_{\mathrm{LSR}} \\
(6)\end{array}$ & $\begin{array}{c}R \\
(7)\end{array}$ & $\begin{array}{c}\tau\left({ }^{13} \mathrm{CO}\right) \\
(8)\end{array}$ & $\begin{array}{c}N\left(\mathrm{H}_{2}\right) \\
(9)\end{array}$ & $\begin{array}{c}M_{\mathrm{LTE}} \\
(10)\end{array}$ & $\begin{array}{l}M_{\mathrm{vir}} \\
(11)\end{array}$ \\
\hline $16 a$ & 105.11 & -38.03 & 0.7 & 1.7 & -11.4 & 0.06 & 0.11 & 4.0 & 0.08 & 34.6 \\
\hline $16 \mathrm{~b}$ & 105.12 & -37.80 & 0.7 & 1.0 & -13.1 & 0.07 & 0.12 & 3.6 & 0.09 & 13.5 \\
\hline $19 \mathrm{a}$ & 105.54 & -38.63 & 0.8 & 1.1 & -5.5 & 0.09 & 0.14 & 2.5 & 0.17 & 23.2 \\
\hline $19 \mathrm{~b}$ & 105.77 & -38.37 & 2.3 & 1.7 & -4.0 & 0.21 & 0.46 & 16.6 & 1.41 & 129.8 \\
\hline 20 & 103.70 & -39.33 & 1.2 & 1.5 & -9.7 & 0.13 & 0.22 & 10.0 & 0.56 & 57.9 \\
\hline 22 & 106.93 & -36.40 & 0.7 & 2.1 & -9.4 & 0.05 & 0.13 & 3.7 & 0.06 & 45.2 \\
\hline 26 & 107.87 & -53.93 & 0.3 & 1.8 & -7.7 & 0.07 & 0.13 & 2.3 & 0.09 & 46.4 \\
\hline 27 & 108.10 & -53.77 & 0.8 & 3.1 & -6.2 & 0.09 & 0.13 & 3.7 & 0.21 & 176.1 \\
\hline $29 \mathrm{a}$ & 107.82 & -51.70 & 1.2 & 1.6 & -5.0 & 0.08 & 0.21 & 7.0 & 0.21 & 40.7 \\
\hline $29 b$ & 108.78 & -52.63 & 1.4 & 2.0 & -4.6 & 0.14 & 0.26 & 11.1 & 0.87 & 120.6 \\
\hline $29 \mathrm{c}$ & 108.84 & -52.03 & 1.2 & 0.7 & -6.2 & 0.11 & 0.21 & 5.9 & 0.29 & 10.6 \\
\hline $29 \mathrm{~d}$ & 108.89 & -52.17 & 1.3 & 1.1 & -6.9 & 0.08 & 0.23 & 3.8 & 0.13 & 18.4 \\
\hline $29 \mathrm{e}$ & 109.00 & -52.40 & 1.1 & 2.1 & -7.8 & 0.12 & 0.20 & 6.0 & 0.41 & 110.6 \\
\hline $29 f$ & 109.00 & -52.13 & 0.9 & 1.6 & -1.9 & 0.04 & 0.15 & 5.8 & 0.05 & 22.5 \\
\hline $29 \mathrm{~g}$ & 109.16 & -51.87 & 1.0 & 1.7 & -5.8 & 0.10 & 0.17 & 2.3 & 0.25 & 57.6 \\
\hline $29 \mathrm{~h}$ & 109.53 & -51.17 & 0.8 & 0.9 & -6.5 & 0.05 & 0.15 & 2.9 & 0.04 & 8.7 \\
\hline $29 \mathrm{i}$ & 109.64 & -51.57 & 0.3 & 2.4 & -7.0 & 0.05 & 0.12 & 3.2 & 0.04 & 59.7 \\
\hline $29 \mathrm{j}$ & 109.80 & -51.37 & 0.4 & 1.5 & -6.7 & 0.05 & 0.14 & 2.5 & 0.04 & 22.3 \\
\hline $33 a$ & 109.17 & -37.60 & 0.8 & 1.0 & -4.4 & 0.05 & 0.13 & 2.4 & 0.05 & 10.7 \\
\hline $33 \mathrm{~b}$ & 109.21 & -37.80 & 0.9 & 0.9 & -4.1 & 0.06 & 0.16 & 4.7 & 0.08 & 10.6 \\
\hline $33 \mathrm{c}$ & 109.34 & -37.80 & 0.7 & 0.7 & -4.5 & 0.05 & 0.13 & 3.2 & 0.05 & 4.7 \\
\hline 35 & 109.72 & -38.27 & 0.9 & 0.7 & -8.1 & 0.05 & 0.16 & 3.2 & 0.05 & 5.1 \\
\hline 38 & 109.90 & -50.73 & 1.1 & 1.8 & -7.7 & 0.11 & 0.19 & 10.5 & 0.48 & 75.3 \\
\hline $41 \mathrm{a}$ & 110.11 & -41.27 & 1.0 & 2.9 & -5.6 & 0.11 & 0.23 & 10.1 & 0.41 & 197.4 \\
\hline $41 b$ & 110.19 & -41.07 & 0.6 & 2.0 & -7.6 & 0.04 & 0.10 & 2.3 & 0.03 & 33.1 \\
\hline 43 & 111.12 & -41.00 & 0.7 & 1.1 & -5.6 & 0.04 & 0.12 & 4.3 & 0.03 & 9.4 \\
\hline 46 & 112.08 & -39.87 & 0.8 & 0.9 & -5.1 & 0.06 & 0.14 & 3.7 & 0.07 & 9.3 \\
\hline 55 & 113.17 & -52.03 & 1.2 & 0.7 & -7.2 & 0.11 & 0.22 & 4.3 & 0.24 & 10.6 \\
\hline 56 & 113.17 & -42.60 & 0.8 & 0.9 & -10.9 & 0.04 & 0.14 & 3.7 & 0.04 & 6.5 \\
\hline
\end{tabular}


Table 2 - Continued

\begin{tabular}{lcccccccccc}
\hline \hline & & & & & & & & & & \\
No. & $l$ & $b$ & $T_{\mathrm{R}}^{*}$ & $\Delta V$ & $V_{\mathrm{LSR}}$ & $R$ & $\tau\left({ }^{13} \mathrm{CO}\right)$ & $N\left(\mathrm{H}_{2}\right)$ & $M_{\mathrm{LTE}}$ & $M_{\text {vir }}$ \\
$(1)$ & $(2)$ & $(3)$ & $(4)$ & $(5)$ & $(6)$ & $(7)$ & $(8)$ & $(9)$ & $(10)$ & $(11)$ \\
\hline 60 & 114.32 & -51.70 & 0.8 & 1.2 & -9.7 & 0.08 & 0.15 & 4.8 & 0.19 & 24.9 \\
$71 \mathrm{a}$ & 115.49 & -44.40 & 0.9 & 1.0 & -3.5 & 0.10 & 0.16 & 3.7 & 0.20 & 22.2 \\
$71 \mathrm{~b}$ & 116.21 & -44.97 & 1.2 & 1.6 & -3.9 & 0.18 & 0.22 & 9.1 & 1.04 & 93.9 \\
78 & 118.19 & -52.70 & 1.5 & 1.2 & -8.1 & 0.12 & 0.28 & 9.6 & 0.53 & 36.7 \\
\hline
\end{tabular}

Note - Col. (1) : Cloud number of ${ }^{13} \mathrm{CO}$ taken from that of ${ }^{12} \mathrm{CO}$ cloud with which the ${ }^{13} \mathrm{CO}$ cloud is associated. If plural ${ }^{13} \mathrm{CO}$ clouds are associated with one ${ }^{12} \mathrm{CO}$ cloud, a sequential alphabet is added, Col. (2)-(3) : Cloud peak $(l, b)$ position in degree, Col. (4) : Peak temperature in K, Col. (5) : Line width of the composite spectrum in $\mathrm{km} \mathrm{s}^{-1}$, Col. (6) : Peak velocity of the composite spectrum in $\mathrm{km} \mathrm{s}^{-1}$, Col. (7) : Radius of the molecular cloud in pc, Col. (8) : Optical depth of ${ }^{13} \mathrm{CO}$, Col. (9) : Column density of peak position in $10^{20} \mathrm{~cm}^{-2}$, Col. (10) : Mass of the molecular cloud assuming the LTE in $M_{\odot}$, Col. (11) : Virial mass of the molecular cloud in $M_{\odot}$. Col. (4) to (6) are derived by using a single Gaussiun fitting. 